\title{
FLUCTUATIONS FOR ANALYTIC TEST FUNCTIONS IN THE SINGLE RING THEOREM
}

\author{
FLORENT BENAYCH-GEORGES AND JEAN ROCHET
}

\begin{abstract}
We consider a non-Hermitian random matrix $\mathbf{A}$ whose distribution is invariant under the left and right actions of the unitary group. The so-called Single Ring Theorem, proved by Guionnet, Krishnapur and Zeitouni [31], states that the empirical eigenvalue distribution of $\mathbf{A}$ converges to a limit measure supported by an annulus $S$. In this text, we establish the convergence in distribution of random variables of the type $\operatorname{Tr}(f(\mathbf{A}) \mathbf{M})$ where $f$ is analytic on $S$ and the Frobenius norm of $\mathbf{M}$ has order $\sqrt{N}$. As corollaries, we obtain central limit theorems for linear spectral statistics of $\mathbf{A}$ (for analytic test functions) and for finite rank projections of $f(\mathbf{A})$ (like matrix entries). As an application, we locate outliers in multiplicative perturbations of $\mathbf{A}$.
\end{abstract}

\section{INTRODUCTION}

The Single Ring Theorem, by Guionnet, Krishnapur and Zeitouni [31], describes the empirical distribution of the eigenvalues of a large generic matrix with prescribed singular values, i.e. an $N \times N$ matrix of the form $\mathbf{A}=\mathbf{U T V}$, with $\mathbf{U}, \mathbf{V}$ some independent Haardistributed unitary matrices and $\mathbf{T}$ a deterministic matrix whose singular values are the ones prescribed. More precisely, under some technical hypotheses, as the dimension $N$ tends to infinity, if the empirical distribution of the singular values of $\mathbf{A}$ converges to a compactly supported limit measure $\Theta$ on the real line, then the empirical eigenvalues distribution of $\mathbf{A}$ converges to a limit measure $\mu$ on the complex plane which depends only on $\Theta$. The limit measure $\mu$ (see Figure $1(\mathrm{a})$ ) is rotationally invariant in $\mathbb{C}$ and its support is the annulus $S:=\{z \in \mathbb{C} ; a \leq|z| \leq b\}$, with $a, b \geq 0$ such that

$$
a^{-2}=\int x^{-2} \mathrm{~d} \Theta(x) \quad \text { and } \quad b^{2}=\int x^{2} \mathrm{~d} \Theta(x) .
$$

In this text, we consider such a matrix $\mathbf{A}$ and we study (Theorem 2.4) the joint weak convergence, as $N \rightarrow \infty$, of random variables of the type

$$
\operatorname{Tr}(f(\mathbf{A}) \mathbf{M}),
$$

2000 Mathematics Subject Classification. 60B20;15B52;60F05;46L54.

Key words and phrases. Random matrices, Gaussian fluctuations, Single Ring Theorem, Weingarten calculus, Haar measure.

FBG and JR: MAP5, Université Paris Descartes, 45, rue des Saints-Pères 75270 Paris Cedex 06, France. florent.benaych-georges@parisdescartes.fr, jean.rochet@parisdescartes.fr. 
for $f$ an analytic function on the annulus $S$ whose Laurent series expansion has null constant term and $\mathbf{M}$ a deterministic $N \times N$ matrix satisfying some limit conditions. These limit conditions (see (2)) allow to consider both:

- fluctuations, around their limits as predicted by the Single Ring Theorem, of linear spectral statistics of $\mathbf{A}$ (for $\mathbf{M}=\mathbf{I}$ ):

$$
\operatorname{Tr} f(\mathbf{A})=\sum_{i=1}^{N} f\left(\lambda_{i}\right),
$$

where $\lambda_{1}, \ldots, \lambda_{N}$ denote the eigenvalues of $\mathbf{A}$,

- finite rank projections of $f(\mathbf{A})$ (for $\mathbf{M}=\sqrt{N} \times($ a matrix with bounded rank)), like the matrix entries of $f(\mathbf{A})$.

Let us present both of these directions with more details.

1.1. Linear spectral statistics of A. As far as Hermitian random matrices are concerned, linear spectral statistics fluctuations usually come right after the macroscopic behavior, with the microscopic one, in the natural questions that arise (see e.g., among the wide literature on the subject, $[34,37,54,33,6,5,39,53,1,39,4,22,13])$. For unitary or orthogonal matrices, also, many results have been proved (see e.g. the results of Diaconis et al in [27, 28], the ones of Soshnikov in [55] or the ones of Lévy and Maïda in [38]). For non-Hermitian matrices, established results are way less numerous: the first one was [51], by Rider and Silverstein, for analytic test functions of matrices with i.i.d. entries, then came the paper [52] by Rider and Virág, who managed, thanks to the explicit determinantal structure of the correlation functions of the Ginibre ensemble, to study the fluctuations of linear spectral statistics of such matrices for $\mathcal{C}^{1}$ test functions. Recently, in [46], O'Rourke and Renfrew studied the fluctuations of linear spectral statistics of elliptic matrices for analytic test functions and, in [36, 21], Cébron and Kemp used a dynamical approach to study such fluctations on $\mathbb{G L}_{N}$. The reason why, except for the breakthrough of Rider and Virág in [52], many results are limited to analytic test functions is that when non-normal matrices are concerned, functional calculus makes sense only for analytic functions: if one denotes by $\lambda_{1}, \ldots, \lambda_{N}$ the eigenvalues of a non-Hermitian matrix $\mathbf{A}$, one can estimate $\sum_{i=1}^{N} f\left(\lambda_{i}\right)$ out of the numbers $\operatorname{Tr} \mathbf{A}^{k}$ or $\operatorname{Tr}\left((z-\mathbf{A})^{-1}\right)$ only when $f$ is analytic. For a $\mathcal{C}^{2}$ test function $f$, one relies on the explicit joint distribution of the $\lambda_{i}$ 's or on Girko's so-called Hermitization technique, which expresses the empirical spectral measure of $\mathbf{A}$ as the Laplacian of the function $z \longmapsto \log |\operatorname{det}(z-\mathbf{A})|$ (see e.g. [30, 18]). This is a way more difficult problem, which we consider in a forthcoming project.

In this text, as a corollary of our main theorem, we prove that for $\mathbf{A}=\mathbf{U T V}$ an $N \times N$ matrix of the type introduced above and $f$ an analytic function on a neighborhood of the limit support $S$ of the empirical eigenvalue distribution of $\mathbf{A}$, the random variable

$$
\operatorname{Tr} f(\mathbf{A})-\mathbb{E} f(\mathbf{A})
$$


converges in distribution, as $N \rightarrow \infty$, to a centered complex Gaussian random variable with a given covariance matrix (see Corollary 2.8). This is a first step in the study of the noise in the Single Ring Theorem. We notice a quite common fact in random matrix theory: the random variable

$$
\operatorname{Tr} f(\mathbf{A})-\mathbb{E} \operatorname{Tr} f(\mathbf{A})=\sum_{i=1}^{N} f\left(\lambda_{i}\right)-\mathbb{E} f\left(\lambda_{i}\right)
$$

does not need to be renormalized to have a limit in distribution, which reflects the eigenvalue repulsion phenomenon (indeed, would the $\lambda_{i}$ 's have been i.i.d., this random variable would have had order $\sqrt{N})$.

Next, two corollaries are given (Corollaries 2.12 and 2.14), one about the Bergman kernel and the resolvant and one about the log-correlated limit distribution of the characteristic polynomial out of the support.

It should be noted that the class of test functions studied - $f$ analytic on a neighborhood of the annulus where the eigenvalues $\lambda_{i}$ locate asymptotically - is not rich enough to fully characterize the fluctuations the spectrum. For example, not all smooth functions on the annulus can be approximated by analytic functions. Thus while these results do give insight into the fluctuations, the full study of the fluctuations would have to go beyond the realm of analytic test functions.

1.2. Finite rank projections and matrix entries. A century ago, in 1906, Émile Borel proved in [19] that, for a uniformly distributed point $\left(X_{1}, \ldots, X_{N}\right)$ on the unit Euclidian sphere $\mathbb{S}^{N-1}$, the scaled first coordinate $\sqrt{N} X_{1}$ converges weakly to the Gaussian distribution as the dimension $N$ tends to infinity. As explained in the introduction of the paper [3] of Diaconis et al., this means that the features of the "microcanonical" ensemble in a certain model for statistical mechanics (uniform measure on the sphere) are captured by the "canonical" ensemble (Gaussian measure). Since then, a long list of further-reaching results about the asymptotic normality of entries of random orthogonal or unitary matrices have been obtained (see e.g. [3, 40, 23, 26, 35, 10, 12]).

In this text, as a corollary of our main theorem, we prove that for $\mathbf{A}=\mathbf{U T V}$ an $N \times$ $N$ matrix of the type introduced above, $f(z)=\sum_{n \in \mathbb{Z}} a_{n} z^{n}$ an analytic function on a neighborhood of the limit support $S$ of the empirical eigenvalue distribution of $\mathbf{A}$ and $\mathbf{a}, \mathbf{b}$ some unit column vectors, the random variables of the type

$$
\sqrt{N}\left(\mathbf{b}^{*} f(\mathbf{A}) \mathbf{a}-a_{0} \mathbf{b}^{*} \mathbf{a}\right),
$$

which can be seen as particular cases of the variables of the type $\operatorname{Tr}(f(\mathbf{A}) \mathbf{M})-\mathbb{E} \operatorname{Tr}(f(\mathbf{A}) \mathbf{M})$ for $M=\sqrt{N} \mathbf{a b}^{*}$, converge in joint distribution, as $N \rightarrow \infty$, to centered complex Gaussian random variables with a given covariance matrix (see Corollary 2.16). This allows for example to consider matrix entries of $f(\mathbf{A})$, in the vein of the works of Soshnikov et al. for Wigner matrices in [47, 50] (see Corollary 2.19 and Remark 2.20). It also applies to 
the study of finite rank perturbations of $\mathbf{A}$ of multiplicative type: the BBP phase transition (named after the authors of the seminal paper [7]) is well understood for additive or multiplicative perturbations $(\widetilde{\mathbf{A}}=\mathbf{A}+\mathbf{P}$ or $\widetilde{\mathbf{A}}=\mathbf{A}(\mathbf{I}+\mathbf{P}))$ of general Hermitian models (see [48, 20, 14] or [7, 15]), for additive perturbations of various non-Hermitian models (see $[56,16,45,17]$ ), but multiplicative perturbations of non-Hermitian models were so far unexplored. In Remark 2.17 and Figure 1, we explain briefly how our results allow to enlighten a BBP transition for such perturbations.

1.3. Organisation of the paper and proofs. In the next section, we state our main theorem (Theorem 2.4) and its corollaries. The rest of the paper is devoted to the proof of Theorem 2.4, to the proof of Corollary 2.14 and to the appendix.

Theorem 2.4 is proved in three steps. First, we do a cut-off approximation to replace the analytic functions $f$ in the random variables $\operatorname{Tr}(f(\mathbf{A}) \mathbf{M})$ by polynomials. The estimation of the error term in this cut-off is far from obvious relies on non-asymptotic estimates from [11] and [16]. Then, to prove the convergence to Gaussian random variables, we perform a moment calculation, using Weingarten calculus (for the asymptotic fine moments of Haardistributed unitary matrices). Weingarten calculus is the theory, due essentially to Collins and Śniady, of the joint moments of entries of Haar-distributed unitary matrices. We summarize the necessary ingredients of the theory in Appendix A. The third step is the computation of the limit covariance.

\section{MAin Result}

Let $\mathbf{A}$ be a random $N \times N$ matrix implicitly depending on $N$ such that $\mathbf{A}=\mathbf{U T V}$, with $\mathbf{U}, \mathbf{V}, \mathbf{T}$ independent and $\mathbf{U}, \mathbf{V}$ Haar-distributed on the unitary group. We make the following hypotheses on $\mathbf{T}$ :

Assumption 2.1. As $N \rightarrow \infty$, the sequence $\left(N^{-1} \operatorname{Tr} \mathbf{T} \mathbf{T}^{*}\right)^{1 / 2}$ converges in probability to a deterministic limit $b>0$ and there is $M<\infty$ such that with probability tending to one, $\|\mathbf{T}\|_{\text {op }} \leq M$.

Assumption 2.2. With the convention $1 / \infty=0$ and $1 / 0=\infty$, the sequence

$$
\left(N^{-1} \operatorname{Tr}\left(\left(\mathbf{T T}^{*}\right)^{-1}\right)\right)^{-1 / 2}
$$

converges in probability to a deterministic limit $a \geq 0$. If $a>0$, we also suppose that there is $M^{\prime}<\infty$ such that with probability tending to one, $\left\|\mathbf{T}^{-1}\right\|_{\text {op }} \leq M^{\prime}$.

The following, seemingly purely technical, assumption, which could possibly be relaxed following Basak and Dembo's approach of [8], is made to control tails of Laurent series but can be removed if the $f_{j}$ 's have finite Laurent expansion, like in Corollary 2.9 or in Remark 2.20. Precisely, we need it to cite some estimates from [32], where they were proved under this assumption. 
Assumption 2.3. There exist a constant $\kappa>0$ such that

$$
\operatorname{Im}(z)>n^{-\kappa} \Longrightarrow N^{-1}\left|\operatorname{Im} \operatorname{Tr}\left(\left(z-\sqrt{\mathbf{T T}^{*}}\right)^{-1}\right)\right| \leq \frac{1}{\kappa}
$$

For $f$ an analytic function on a neighborhood of the annulus

$$
S:=\{z \in \mathbb{C} ; a \leq|z| \leq b\},
$$

the matrix $f(\mathbf{A})$ is well defined with probability tending to one as $N \rightarrow \infty$, as it was proved in $[32,11]$ that the spectrum of $\mathbf{A}$ is contained in any neighborhood of $S$ with probability tending to one. We denote the Laurent series expansion, on $S$, of any such function $f$ by

$$
f(z)=\sum_{n \in \mathbb{Z}} a_{n}(f) z^{n} .
$$

Theorem 2.4. For each $N \geq 1$, let $\mathbf{M}_{1}, \ldots, \mathbf{M}_{k}$ be $N \times N$ deterministic matrices such that for all $i, j$, as $N \rightarrow \infty$,

$$
\frac{1}{N} \operatorname{Tr} \mathbf{M}_{i} \longrightarrow \tau_{i}, \quad \frac{1}{N} \operatorname{Tr} \mathbf{M}_{i} \mathbf{M}_{j}^{*} \longrightarrow \alpha_{i j}, \frac{1}{N} \operatorname{Tr} \mathbf{M}_{i} \mathbf{M}_{j} \longrightarrow \beta_{i j}
$$

Let $f_{1}, \ldots, f_{k}$ be analytic on a neighborhood of $S$. Then, as $N \rightarrow \infty$, the random vector

$$
\left(\operatorname{Tr} f_{j}(\mathbf{A}) \mathbf{M}_{j}-a_{0}\left(f_{j}\right) \operatorname{Tr} \mathbf{M}_{j}\right)_{j=1}^{k}
$$

converges to a centered complex Gaussian vector $\left(\mathcal{G}\left(f_{1}\right), \ldots, \mathcal{G}\left(f_{k}\right)\right)$ whose distribution is defined by

$$
\begin{aligned}
& \mathbb{E} \mathcal{G}\left(f_{i}\right) \mathcal{G}\left(f_{j}\right)=\sum_{n \geq 1}\left((n-1) \tau_{i} \tau_{j}+\beta_{i j}\right)\left(a_{n}\left(f_{i}\right) a_{-n}\left(f_{j}\right)+a_{-n}\left(f_{i}\right) a_{n}\left(f_{j}\right)\right) \\
& \mathbb{E} \mathcal{G}\left(f_{i}\right) \overline{\mathcal{G}\left(f_{j}\right)}=\sum_{n \geq 1}\left((n-1) \tau_{i} \overline{\tau_{j}}+\alpha_{i j}\right)\left(a_{n}\left(f_{i}\right) \overline{a_{n}\left(f_{j}\right)} b^{2 n}+a_{-n}\left(f_{i}\right) \overline{a_{-n}\left(f_{j}\right)} a^{-2 n}\right) .
\end{aligned}
$$

Remark 2.5. In (3),

rewrites

$$
\operatorname{Tr} f_{j}(\mathbf{A}) \mathbf{M}_{j}-a_{0}\left(f_{j}\right) \operatorname{Tr} \mathbf{M}_{j}
$$

$$
\operatorname{Tr} f_{j}(\mathbf{A}) \mathbf{M}_{j}-\mathbb{E} \operatorname{Tr} f_{j}(\mathbf{A}) \mathbf{M}_{j} .
$$

Indeed, $\mathbb{E} f(\mathbf{A})=a_{0} \mathbf{I}$, as a consequence of the fact that for any $n \neq 0$, for any $\theta \in \mathbb{R}$, $\mathbf{A}^{n} \stackrel{\text { law }}{=} \mathrm{e}^{\mathrm{i} \theta} \mathbf{A}^{n}$, which follows from the invariance of the Haar measure.

Remark 2.6. Note that if $a=0$, as the $f_{j}$ 's are analytic on $S$, we have $a_{-n}\left(f_{j}\right)=0$ for each $n \geq 1$ and each $j$, so that the above expression still makes sense. Besides, it seems reasonable to verify that the two series above converge:

$$
\begin{aligned}
\sum_{n \geq 1} n\left|a_{n}\left(f_{i}\right)\right|\left|a_{n}\left(f_{j}\right)\right| b^{2 n} & \leq\left(\max _{n \geq 1}\left|a_{n}\left(f_{j}\right)\right| b^{n}\right) \sum_{n \geq 1} n\left|a_{n}\left(f_{i}\right)\right| b^{n}<\infty \\
\sum_{n \geq 1} n\left|a_{n}\left(f_{i}\right)\right|\left|a_{-n}\left(f_{j}\right)\right| & \leq\left(\max _{n \geq 1}\left|a_{n}\left(f_{i}\right)\right| b^{n}\right) \sum_{n \geq 1} n\left|a_{-n}\left(f_{j}\right)\right| a^{-n}<\infty .
\end{aligned}
$$


Remark 2.7 (Relation to second order freeness). A theory has been developed recently about Gaussian fluctuations (called second order limits) of traces of large random matrices, the most emblematic articles in this theory being [41, 42, 43, 24]. Theorem 2.4 can be compared to some of these results. However, our hypotheses on the matrices we consider are of a different nature than the ones of the previously cited papers, since the convergence of the non commutative distributions is not required here: our hypotheses are satisfied for example by matrices like $\mathbf{M}_{j}=\sqrt{N} \times($ an elementary $N \times N$ matrix $)$, which have no bounded moments of order higher than two.

Our two main applications are the case where the $\mathbf{M}_{j}$ 's are all $\mathbf{I}$ (Corollaries 2.8 and 2.9) and the cases where the $\mathbf{M}_{j}$ 's are $\sqrt{N}$ times matrices with bounded rank and norm, like elementary matrices (Corollaries 2.16 and 2.19). In the case $\mathbf{M}=\mathbf{I}$, we immediately obtain the following corollary about linear spectral statistics of $\mathbf{A}$.

Corollary 2.8. Let $f_{1}, \ldots, f_{k}$ be analytic on a neighborhood of $S$. Then, as $N \rightarrow \infty$, the random vector

$$
\left(\operatorname{Tr} f_{j}(\mathbf{A})-N a_{0}\left(f_{j}\right)\right)_{j=1}^{k}
$$

converges to a centered complex Gaussian vector $\left(\mathcal{G}\left(f_{1}\right), \ldots, \mathcal{G}\left(f_{k}\right)\right)$ such that

$$
\begin{aligned}
\mathbb{E} \mathcal{G}\left(f_{i}\right) \mathcal{G}\left(f_{j}\right) & =\sum_{n \in \mathbb{Z}}|n| a_{n}\left(f_{i}\right) a_{-n}\left(f_{j}\right) \\
\mathbb{E} \mathcal{G}\left(f_{i}\right) \overline{\mathcal{G}\left(f_{j}\right)} & =\sum_{n \geq 1} n\left(a_{n}\left(f_{i}\right) \overline{a_{n}\left(f_{j}\right)} b^{2 n}+a_{-n}\left(f_{i}\right) \overline{a_{-n}\left(f_{j}\right)} a^{-2 n}\right) .
\end{aligned}
$$

For $n \geq 1$, let us define the functions

$$
\varphi_{n}^{ \pm}(z):=\left(\frac{z}{b}\right)^{n} \pm\left(\frac{a}{z}\right)^{n}
$$

These functions (plus the constant one) define a basis of the space of analytic functions on a neighborhood of $S$ and we have the change of basis formula

$$
\sum_{n \in \mathbb{Z}} a_{n} z^{n}=a_{0}+\sum_{n \geq 1} c_{n}^{+} \varphi_{n}^{+}(z)+c_{n}^{-} \varphi_{n}^{-}(z) \Longleftrightarrow \forall n \geq 1,\left(\begin{array}{c}
a_{n} \\
a_{-n}
\end{array}\right)=\left(\begin{array}{cc}
b^{-n} & b^{-n} \\
a^{n} & -a^{n}
\end{array}\right)\left(\begin{array}{c}
c_{n}^{+} \\
c_{n}^{-}
\end{array}\right),
$$

implying that

$$
\sum_{n \geq 1}\left|a_{n}(f)\right|^{2} b^{2 n}+\left|a_{-n}(f)\right|^{2} a^{-2 n}=2 \sum_{n \geq 1}\left|c_{n}^{+}(f)\right|^{2}+\left|c_{n}^{-}(f)\right|^{2} .
$$

Besides, these functions allow to identify the underlying white noise in Theorem 2.4 (we only state it here in the case $\mathbf{M}_{j}=\mathbf{I}$, but this of course extends to the case of general $\mathbf{M}_{j}$ 's, allowing for example to state analogous results for the matrix entries).

Corollary 2.9 (Underlying white noise). The finite dimensional marginal distributions of

$$
\left(\operatorname{Tr} \varphi_{n}^{+}(\mathbf{A})\right)_{n \geq 1} \bigcup\left(\operatorname{Tr} \varphi_{n}^{-}(\mathbf{A})\right)_{n \geq 1}
$$


converge to the ones of a collection $\left(\mathcal{G}_{n}^{+}\right)_{n \geq 1} \cup\left(\mathcal{G}_{n}^{-}\right)_{n \geq 1}$ of independent centered complex Gaussian random variables satisfying

$$
\mathbb{E}\left|\mathcal{G}_{n}^{ \pm}\right|^{2}=2 \quad ; \quad \mathbb{E}\left(\mathcal{G}_{n}^{ \pm}\right)^{2}= \pm 2 n(a / b)^{n} .
$$

Remark 2.10 (Ginibre matrices). In the particular case where $\mathbf{A}$ is a Ginibre matrix (i.e. with i.i.d. entries with law $\left.\mathcal{N}_{\mathbb{C}}\left(0, N^{-1}\right)\right)$, we reproduce the result of Rider and Silverstein [51], noticing that in this case $a=0$ and $b=1$, so that $a_{n}(f)=0$ when $n<0$ and $\mathbb{E} \mathcal{G}\left(f_{i}\right) \mathcal{G}\left(f_{j}\right)=0$, and, for $\mathrm{d} m(z)$ the Lebesgue measure on $\mathbb{C}$,

$$
\begin{aligned}
& \frac{1}{\pi} \int_{|z|<1} \frac{\partial}{\partial z} f_{i}(z) \overline{\frac{\partial}{\partial z} f_{j}(z)} \mathrm{d} m(z) \\
= & \frac{1}{\pi} \int_{|z|<1}-\frac{1}{4 \pi^{2}} \oint_{\operatorname{Circle}(1+\varepsilon)} \oint_{\operatorname{Circle}(1+\varepsilon)} \frac{f_{i}\left(\xi_{1}\right)}{\left(\xi_{1}-z\right)^{2}} \frac{\overline{f_{j}\left(\xi_{2}\right)}}{\left(\overline{\xi_{2}}-\bar{z}\right)^{2}} \mathrm{~d} \xi_{1} \mathrm{~d} \xi_{2} \mathrm{~d} m(z) \\
= & -\frac{1}{4 \pi^{2}} \oint_{\operatorname{Circle}(1+\varepsilon)} \oint_{\operatorname{Circle}(1+\varepsilon)} \frac{f_{i}\left(\xi_{1}\right) \overline{f_{j}\left(\xi_{2}\right)}}{\xi_{1}^{2}{\overline{\xi_{2}}}^{2}} \frac{1}{\pi} \int_{|z|<1} \sum_{n, n^{\prime} \geq 1} n n^{\prime}\left(\frac{z}{\xi_{1}}\right)^{n-1}\left(\frac{\bar{z}}{\bar{\xi}_{2}}\right)^{n^{\prime}-1} \mathrm{~d} m(z) \mathrm{d} \xi_{1} \mathrm{~d} \xi_{2} \\
= & -\frac{1}{4 \pi^{2}} \oint_{\operatorname{Circle}(1+\varepsilon)} \oint_{\operatorname{Circle}(1+\varepsilon)} f_{i}\left(\xi_{1}\right) \overline{f_{j}\left(\xi_{2}\right)} \sum_{n \geq 1} n\left(\xi_{1} \overline{\xi_{2}}\right)^{-n-1} \mathrm{~d} \xi_{1} \mathrm{~d} \xi_{2} \\
= & \sum_{n \geq 1} n a_{n}\left(f_{i}\right) \overline{a_{n}\left(f_{j}\right)}
\end{aligned}
$$

Remark 2.11. If $\mathbf{T}=\mathbf{I}$, and the $f_{k}$ 's are polynomial, we reproduce a result of Diaconis and Shahshahani [27, Theorem 1] on the limit joint distribution of

$$
\left(\operatorname{Tr}\left(\mathbf{U}^{k}\right)\right)_{k=1}^{n} \text {, }
$$

where $\mathbf{U}$ is Haar-distributed. Actually, the Corollary 2.8 is slightly stronger, since the result holds for $\mathbf{A}=\mathbf{U T}$ or $\mathbf{A}=\mathbf{U T V}$ as long as $\mathbf{T}$ satisfies

$$
\lim _{N \rightarrow \infty} \frac{1}{N} \operatorname{Tr}\left(\mathbf{T} \mathbf{T}^{*}\right)=\lim _{N \rightarrow \infty} \frac{1}{N} \operatorname{Tr}\left(\left(\mathbf{T} \mathbf{T}^{*}\right)^{-1}\right)=1
$$

in which case $\mathbf{A}$ may be seen as a multiplicative perturbation of $\mathbf{U}$. Indeed, (4) implies that all singular values of $\mathbf{T}$ are close to 1 . The matrix $\mathbf{T}$ satisfies the condition (4) for example if it is diagonal and all its diagonal coefficients are equal to 1 except $o(N)$ of them (which stay away from 0 and $\infty$ ).

Corollary 2.12 (Bergman kernel and resolvant). The random process

$$
\left(\operatorname{Tr}(z-\mathbf{A})^{-1}\right)_{|z|<a} \cup\left(\operatorname{Tr}(z-\mathbf{A})^{-1}\right)_{|z|>b}
$$

converges, for the finite-dimensional distributions, to a centered complex Gaussian process

$$
\left(\mathcal{G}_{z}\right)_{|z|<a} \cup\left(\mathcal{H}_{z}\right)_{|z|>b}
$$

with covariance defined by

$$
\mathbb{E} \mathcal{G}_{z} \overline{\mathcal{G}_{z^{\prime}}}=\frac{b^{2}}{\left(b^{2}-z \overline{z^{\prime}}\right)^{2}}, \quad \mathbb{E} \mathcal{H}_{z} \overline{\mathcal{H}_{z^{\prime}}}=\frac{a^{2}}{\left(a^{2}-z \overline{z^{\prime}}\right)^{2}}, \quad \mathbb{E} \mathcal{G}_{z} \mathcal{H}_{z^{\prime}}=-\frac{1}{\left(z^{\prime}-z\right)^{2}}
$$


and by the fact that

$$
\forall \theta \in \mathbb{R}, \quad\left(\mathrm{e}^{-\mathrm{i} \theta} \mathcal{G}_{z}\right)_{|z|<a} \cup\left(\mathrm{e}^{\mathrm{i} \theta} \mathcal{H}_{z}\right)_{|z|>b} \stackrel{\text { law }}{=}\left(\mathcal{G}_{z}\right)_{|z|<a} \cup\left(\mathcal{H}_{z}\right)_{|z|>b} .
$$

Remark 2.13. The kernel of the limit Gaussian analytic function, in the previous corollary, is, up to a constant factor, the Bergman kernel (see [9, 49]).

Corollary 2.14 (Characteristic polynomial out of the support). The random process

$$
(\log |\operatorname{det}(z-\mathbf{A})|-\operatorname{Tr} \log \mathbf{T})_{|z|<a} \cup(\log |\operatorname{det}(z-\mathbf{A})|-N \log |z|)_{|z|>b}
$$

converges, for the finite-dimensional distributions, to a centered real Gaussian process

$$
\left(\mathcal{G}_{z}\right)_{|z|<a} \cup\left(\mathcal{H}_{z}\right)_{|z|>b}
$$

with covariance defined by

$2 \mathbb{E} \mathcal{G}_{z} \mathcal{G}_{z^{\prime}}=-\log \left|1-\frac{z \overline{z^{\prime}}}{a^{2}}\right|, \quad 2 \mathbb{E} \mathcal{H}_{z} \mathcal{H}_{z^{\prime}}=-\log \left|1-\frac{b^{2}}{z \overline{z^{\prime}}}\right|, \quad 2 \mathbb{E} \mathcal{G}_{z} \mathcal{H}_{z^{\prime}}=-\log \left|1-\frac{z}{z^{\prime}}\right|$.

Remark 2.15. As $z \neq z^{\prime}$ both tend to the same point on the boundary of $S$, the above covariances are equivalent to $-\log \left|z-z^{\prime}\right|$. In the light of the log-correlation approach to the Gaussian Free Field (see [29]), it supports the idea that on the limit support $S$, the characteristic polynomial of $\mathbf{A}$ should tend to an object related to the Gaussian Free Field, as for Ginibre matrices (see Corollary 2 of [52]). It would be interesting to see to what extent such a convergence depends on the hypotheses made on the precise distribution of the singular values of $\mathbf{T}$.

In the case $\mathbf{M}_{j}=\sqrt{N} \mathbf{a}_{j} \mathbf{b}_{j}^{*}$, we immediately obtain the following corollary:

Corollary 2.16. For each $N \geq 1$, let $\mathbf{a}_{1}, \mathbf{b}_{1}, \ldots, \mathbf{a}_{k}, \mathbf{b}_{k}$ be deterministic column vectors with size $N$ such that for all $i, j$, as $N \rightarrow \infty$,

$$
\mathbf{a}_{i}^{*} \mathbf{a}_{j} \longrightarrow \kappa_{i j}^{\mathrm{a}, \mathrm{a}} \in \mathbb{C} \quad ; \quad \mathbf{b}_{i}^{*} \mathbf{a}_{j} \longrightarrow \kappa_{i j}^{\mathrm{b}, \mathrm{a}} \in \mathbb{C} \quad ; \quad \mathbf{b}_{i}^{*} \mathbf{b}_{j} \longrightarrow \kappa_{i j}^{\mathrm{b}, \mathrm{b}} \in \mathbb{C}
$$

Let $f_{1}, \ldots, f_{k}$ be analytic on a neighborhood of $S$. Then, as $N \rightarrow \infty$, the random vector

$$
\sqrt{N}\left(\mathbf{b}_{j}^{*} f_{j}(\mathbf{A}) \mathbf{a}_{j}-\mathbf{b}_{j}^{*} \mathbf{a}_{j} a_{0}\left(f_{j}\right)\right)_{j=1}^{k}
$$

converges to a centered complex Gaussian vector $\left(\mathcal{G}\left(f_{1}\right), \ldots, \mathcal{G}\left(f_{k}\right)\right)$ such that

$$
\begin{aligned}
& \mathbb{E} \mathcal{G}\left(f_{i}\right) \mathcal{G}\left(f_{j}\right)=\sum_{n \geq 1} \kappa_{j i}^{\mathrm{a}, \mathrm{a}} \kappa_{i j}^{\mathrm{b}, \mathrm{b}}\left(a_{n}\left(f_{i}\right) a_{-n}\left(f_{j}\right)+a_{-n}\left(f_{i}\right) a_{n}\left(f_{j}\right)\right) \\
& \mathbb{E} \mathcal{G}\left(f_{i}\right) \overline{\mathcal{G}\left(f_{j}\right)}=\sum_{n \geq 1} \kappa_{j i}^{\mathrm{b}, \mathrm{a}} \kappa_{i j}^{\mathrm{b}, \mathrm{a}}\left(a_{n}\left(f_{i}\right) \overline{a_{n}\left(f_{j}\right)} b^{2 n}+a_{-n}\left(f_{i}\right) \overline{a_{-n}\left(f_{j}\right)} a^{-2 n}\right) .
\end{aligned}
$$

Remark 2.17 (Application to multiplicative finite rank perturbations of A). The previous corollary has several applications to the study of the outliers of spiked models related to the Single Ring Theorem. It allows for example to understand easily, using the techniques developed in [16], the impact of multiplicative finite rank perturbations on the spectrum 
of $\mathbf{A}$ (whereas only additive perturbations had been studied so far). For example, one can deduce from this corollary that for $\mathbf{P}$ a deterministic matrix with bounded operator norm and rank one, if one defines $\widetilde{\mathbf{A}}:=\mathbf{A}(\mathbf{I}+\mathbf{P})$ and $\hat{\mathbf{A}}:=\mathbf{A}(\mathbf{I}+\mathbf{A P})$, then

- the matrix $\widetilde{\mathbf{A}}$ has no outlier (i.e. the support of its spectrum still converges to $S$ ),

- the matrix $\hat{\mathbf{A}}$ has no outlier with modulus $>b$, but each eigenvalue $\lambda$ of $\mathbf{P}$ such that $|\lambda|>a^{-1}$ gives rise to an outlier of $\hat{\mathbf{A}}$ located approximately at $-\lambda^{-1}$ (besides, when the multiplicity of $\lambda$ as an eigenvalue of $\mathbf{P}$ is 1 , the fluctuations of the outlier around $-\lambda^{-1}$ are Gaussian and with order $N^{-1 / 2}$ ).

This phenomenon is illustrated by Figure 1.

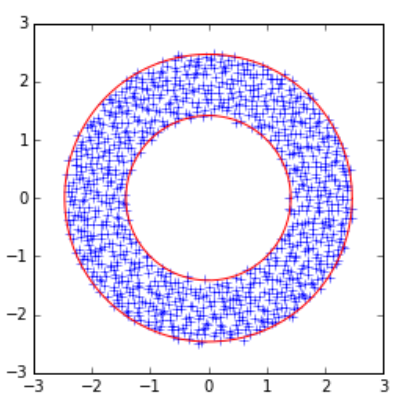

(a) Spectrum of $\mathbf{A}$

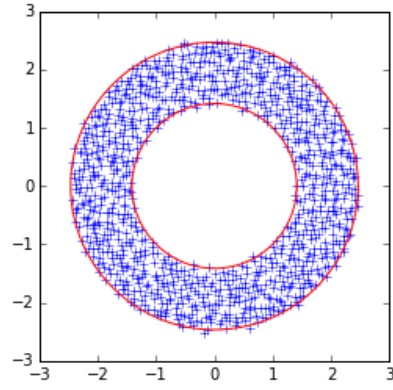

(b) Spectrum of $\widetilde{\mathbf{A}}:=$ $\mathbf{A}(\mathbf{I}+\mathbf{P})$

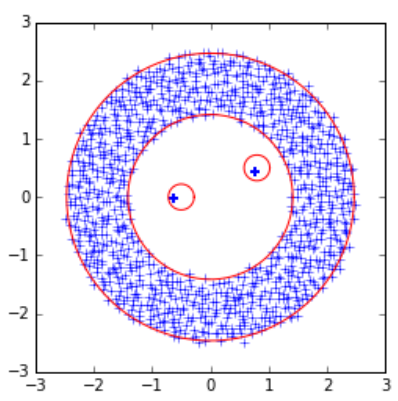

(c) Spectrum of $\hat{\mathbf{A}}:=$ $\mathbf{A}(\mathbf{I}+\mathbf{A P})$ (small circles are centered at the theoretical limit locations of the outliers)

FIGURE 1. Outliers/lack of outliers for multiplicative perturbations: simulation realized with a single $10^{3} \times 10^{3}$ matrix $\mathbf{A}=\mathbf{U T V}$ when the singular values of $\mathbf{T}$ are uniformly distributed on $[0.5,4]$ and $\mathbf{P}=\operatorname{diag}(-2,(0.8+$ $\left.0.5 \mathrm{i})^{-1}, 1 / 3,0, \ldots, 0\right)$. As predicted, none of these matrices has any outlier outside the outer circle, nor do the two first ones inside the inner circle, but $\hat{\mathbf{A}}$ has two outliers inside the inner circle, close to the predicted locations.

To state the next corollary, let us first give the definition of Gaussian elliptic matrices.

Definition 2.18 (Gaussian elliptic matrices). Let $\rho \in \mathbb{C}$ such that $|\rho| \leq 1$. A Gaussian elliptic matrix with parameter $\rho$ is an $N \times N$ Gaussian centered complex random matrix $\mathbf{X}=\left(x_{i j}\right)$ satisfying :

(1) the random vectors $\left(x_{i j}, x_{j i}\right)_{i \leq j}$ are independent,

(2) $\forall i, \mathbb{E}\left|x_{i i}\right|^{2}=1$ and $\mathbb{E} x_{i i}^{2}=\rho$,

(3) $\forall i \neq j, \mathbb{E}\left|x_{i j}\right|^{2}=1, \mathbb{E} x_{i j}^{2}=0, \mathbb{E} x_{i j} x_{j i}=\rho$ and $\mathbb{E} x_{i j} \overline{x_{j i}}=0$. 
This matrix ensemble was introduced by Girko in [30], and its name is due to the fact that its empirical eigenvalue distribution is the uniform distribution inside an ellipse. In the case where $\rho=0$ (resp. $\rho=1$ ), we get a Ginibre (resp. GUE) matrix.

Corollary 2.19. Let $f$ be analytic on a neighborhood of $S$ such that

$$
\sum_{n \geq 1}\left|a_{n}(f)\right|^{2} b^{2 n}+\left|a_{-n}(f)\right|^{2} a^{-2 n}=1
$$

Let $k$ be a fixed positive integer and let $I=I(N)$ be a (possibly $N$-dependent) subset of $\{1, \ldots, N\}$ with cardinality $k$. Let us define the random $k \times k$ matrix

$$
\mathbf{X}_{N}:=\sqrt{N}\left[f(\mathbf{A})_{i j}-a_{0}(f) \delta_{i j}\right]_{(i, j) \in I \times I} .
$$

Then, as $N \rightarrow \infty$, the matrix $\mathbf{X}_{N}$ converges in distribution to a $k \times k$ Gaussian elliptic matrix $\mathbf{X}$ with parameter $\rho$,

$$
\rho:=2 \sum_{n \geq 1} a_{n}(f) a_{-n}(f)
$$

Remark 2.20. a) In the case where $f(z)=z$, we rederive the well-known result that any fixed-size principal submatrix of $\sqrt{N} \mathbf{U}$ converges to a Ginibre matrix (see e.g. $[3,35])$.

b) By Corollary 2.19, the statement of the first part of this remark happens to stay true, up to a constant multiplicative factor, if $\mathbf{U}$ is replaced by $\mathbf{A}=\mathbf{U T V}$ or even by $\mathbf{A}^{n}$ or by $f(\mathbf{A})$ if $f$ is analytic in a neighborhood of the $\operatorname{disc} \bar{B}(0, b)$.

c) It also follows from what precedes that for any $n \geq 1$, any sequence of principal submatrices with fixed size of $\sqrt{N / 2}\left(\mathbf{U}^{n}+\mathbf{U}^{-n}\right)$ and $\sqrt{N / 2}\left(\mathbf{U}^{n}-\mathbf{U}^{-n}\right)$ converge in distribution to a GUE matrix and i times a GUE matrix, both being independent.

\section{Proof of Theorem 2.4}

To avoid having to treat the cases $a>0$ and $a=0$ separately all along the proof, we shall suppose that $a>0$ (the case $a=0$ is more simple, as sums run only on $n \geq 0$ ). Besides, note that by invariance of the Haar measure, the distribution of the random matrix A depends on $\mathbf{T}$ only through its singular values, so we shall suppose that $\mathbf{T}=$ $\operatorname{diag}\left(s_{1}, \ldots, s_{N}\right)$, with $s_{i} \geq 0$. At last, as the limit distributions, in Theorem 2.4, only depend on $\mathbf{T}$ only through the deterministic parameters $a, b$, up to a conditioning, one can suppose that $\mathbf{T}$ is deterministic (and that both $\|\mathbf{T}\|_{\text {op }}$ and $\left\|\mathbf{T}^{-1}\right\|_{\text {op }}$ are uniformly bounded, by Asssumptions 2.1 and 2.2).

3.1. Randomization of the $\mathbf{M}_{j}$ 's. Let us define $\mathbf{W}:=\mathbf{V U}$. The random matrix $\mathbf{W}$ is also Haar-distributed and independent from $\mathbf{V}$. Besides, for each $j$, as $\mathbf{A}=\mathbf{U T V}=$ $\mathbf{V}^{*} \mathbf{W T V}$,

$$
\operatorname{Tr} f_{j}(\mathbf{A}) \mathbf{M}_{j}=\operatorname{Tr} \mathbf{V}^{*} f_{j}(\mathbf{W T}) \mathbf{V} \mathbf{M}_{j}=\operatorname{Tr} f_{j}(\mathbf{W T}) \mathbf{V} \mathbf{M}_{j} \mathbf{V}^{*}
$$


As a consequence, we shall suppose that $\mathbf{A}=\mathbf{U T}$ (instead of $\mathbf{A}=\mathbf{U T V}$ ) and that there is a Haar-distributed random unitary matrix $\mathbf{V}$, independent of $\mathbf{U}$, such that for each $j$, $\mathbf{M}_{j}=\mathbf{V} \widetilde{\mathbf{M}}_{j} \mathbf{V}^{*}$, with $\widetilde{\mathbf{M}}_{1}, \ldots, \widetilde{\mathbf{M}}_{k}$ a collection of deterministic matrices also satisfying (2).

3.2. Tails of the series. Let us first prove that Theorem 2.4 can be deduced from the particular case where there is $n_{0}$ such that for all $n$, we have

$$
|n|>n_{0} \Longrightarrow \forall j=1, \ldots, k, a_{n}\left(f_{j}\right)=0 .
$$

Let $\varepsilon \in(0, a / 2)$ such that the domain of each $f_{j}$ contains the annulus of complex numbers $z$ such that $a-2 \varepsilon \leq|z| \leq b+2 \varepsilon$.

Lemma 3.1. There is a constant $C$ independent of $N$ such that for any $n$ such that $n^{6} \leq N$ and any $j=1, \ldots, k$, we have

$$
\mathbb{E}\left|\operatorname{Tr} \mathbf{A}^{n} \mathbf{M}_{j}\right|^{2} \leq C n^{2}\left(\mathbb{1}_{n \geq 0}(b+\varepsilon)^{2 n}+\mathbb{1}_{n \leq 0}(a-\varepsilon)^{2 n}\right)
$$

Proof. With the notation of Section 3.1, let $\mathbb{E}_{\mathbf{V}}$ denote the expectation with respect to the randomness of $\mathbf{V}$. For each $n \in \mathbb{Z}$ and each $j$, by Lemma A.6, we have

$$
\begin{aligned}
\mathbb{E}_{\mathbf{V}}\left|\operatorname{Tr} \mathbf{A}^{n} \mathbf{M}_{j}\right|^{2}= & \mathbb{E}_{\mathbf{V}} \operatorname{Tr} \mathbf{A}^{n} \mathbf{V} \widetilde{\mathbf{M}}_{j} \mathbf{V}^{*} \operatorname{Tr}\left(\mathbf{A}^{*}\right)^{n} \mathbf{V} \widetilde{\mathbf{M}}_{j}^{*} \mathbf{V}^{*} \\
= & \frac{1}{N^{2}-1}\left(\operatorname{Tr} \mathbf{A}^{n} \operatorname{Tr}\left(\mathbf{A}^{*}\right)^{n} \operatorname{Tr} \widetilde{\mathbf{M}}_{j} \operatorname{Tr} \widetilde{\mathbf{M}}_{j}^{*}+\operatorname{Tr} \mathbf{A}^{n}\left(\mathbf{A}^{*}\right)^{n} \operatorname{Tr} \widetilde{\mathbf{M}}_{j} \widetilde{\mathbf{M}}_{j}^{*}\right) \\
& -\frac{1}{N\left(N^{2}-1\right)}\left(\operatorname{Tr} \mathbf{A}^{n} \operatorname{Tr}\left(\mathbf{A}^{*}\right)^{n} \operatorname{Tr} \widetilde{\mathbf{M}} \widetilde{\mathbf{M}}_{j}^{*}+\operatorname{Tr} \mathbf{A}^{*}\left(\mathbf{A}^{*}\right)^{n} \operatorname{Tr} \widetilde{\mathbf{M}}_{j} \operatorname{Tr} \widetilde{\mathbf{M}}_{j}^{*}\right) \\
\leq & \frac{1}{N^{2}-1}\left(\left|\operatorname{Tr} \mathbf{A}^{n}\right|^{2} \operatorname{Tr} \widetilde{\mathbf{M}}_{j} \operatorname{Tr} \widetilde{\mathbf{M}}_{j}^{*}+\operatorname{Tr} \mathbf{A}^{n}\left(\mathbf{A}^{*}\right)^{n} \operatorname{Tr} \widetilde{\mathbf{M}}_{j} \widetilde{\mathbf{M}}_{j}^{*}\right) \\
\leq & C\left(\left|\operatorname{Tr} \mathbf{A}^{n}\right|^{2}+N^{-1} \operatorname{Tr} \mathbf{A}^{n}\left(\mathbf{A}^{*}\right)^{n}\right),
\end{aligned}
$$

where $C$ is a constant independent of $N$. Then the conclusion follows from Lemma A.5.

Lemma 3.2. There are some constants $C>0$ and $c \in(0,1)$ and a sequence $\mathcal{E}=\mathcal{E}_{N}$ of events such that

$$
\mathbb{P}(\mathcal{E}) \underset{N \rightarrow \infty}{\longrightarrow} 1
$$

and such that for all $N$, all $n_{1} \geq 0$ and all $j=1, \ldots, k$, we have

$$
\mathbb{E}\left|\mathbb{1}_{\mathcal{E}} \sum_{|n|>n_{1}} a_{n}\left(f_{j}\right) \operatorname{Tr}\left(\mathbf{A}^{n} \mathbf{M}_{j}\right)\right| \leq C N(1-c)^{n_{1}}
$$

Proof. By [16, Lem. 3.2], we known that there is a constant $C_{1}$ such that the event

$$
\mathcal{E}=\mathcal{E}_{N}:=\left\{\forall n \geq 0,\left\|\mathbf{A}^{n}\right\|_{\text {op }} \leq C_{1}(b+\varepsilon)^{n}\right\} \cap\left\{\forall n \leq 0,\left\|\mathbf{A}^{n}\right\|_{\text {op }} \leq C_{1}(a-\varepsilon)^{n}\right\}
$$

has probability tending to one. 
Then one concludes easily, noting first that by non-commutative Hölder inequalities (see $[2$, Eq. (A.13)]), we have

$$
\mathbb{1}_{\mathcal{E}}\left|\operatorname{Tr}\left(\mathbf{A}^{n} \mathbf{M}_{j}\right)\right| \leq \begin{cases}C_{1}(b+\varepsilon)^{n} N \sqrt{N^{-1} \operatorname{Tr} \mathbf{M}_{j} \mathbf{M}_{j}^{*}} & \text { if } n \geq 0 \\ C_{1}(a-\varepsilon)^{n} N \sqrt{N^{-1} \operatorname{Tr} \mathbf{M}_{j} \mathbf{M}_{j}^{*}} & \text { if } n \leq 0\end{cases}
$$

and secondly that there is $c \in(0,1)$ such that for each $j$, the sequences

$$
\left(a_{n}\left(f_{j}\right) \frac{(b+\varepsilon)^{n}}{(1-c)^{n}}\right)_{n \geq 0} \quad ; \quad\left(a_{n}\left(f_{j}\right) \frac{(a-\varepsilon)^{n}}{(1-c)^{-n}}\right)_{n \leq 0}
$$

are bounded.

As a consequence of Lemmas 3.1 and 3.2, for any $0<n_{0}<n_{1} \leq N^{1 / 6}$ and any $j=1, \ldots, k$,

$$
\begin{aligned}
\mathbb{E}\left|\mathbb{1}_{\mathcal{E}} \sum_{|n|>n_{0}} a_{n}\left(f_{j}\right) \operatorname{Tr}\left(\mathbf{A}^{n} \mathbf{M}_{j}\right)\right| \leq & \sum_{n_{0}<|n| \leq n_{1}}\left|a_{n}\left(f_{j}\right)\right| \sqrt{\mathbb{P}(\mathcal{E})} \sqrt{\mathbb{E}\left|\operatorname{Tr}\left(\mathbf{A}^{n} \mathbf{M}_{j}\right)\right|^{2}} \\
& +\mathbb{E}\left|\mathbb{1}_{\mathcal{E}} \sum_{|n|>n_{1}} a_{n}\left(f_{j}\right) \operatorname{Tr}\left(\mathbf{A}^{n} \mathbf{M}_{j}\right)\right| \\
\leq & \sum_{n_{0}<|n| \leq n_{1}} C\left|a_{n}\left(f_{j}\right)\right| n^{2}\left(\mathbb{1}_{n \geq 0}(b+\varepsilon)^{2 n}+\mathbb{1}_{n \leq 0}(a-\varepsilon)^{2 n}\right) \\
& +C N(1-c)^{n_{1}}
\end{aligned}
$$

Choosing first $n_{1}=\lfloor A \log N\rfloor$ for $A$ a large enough constant and then using the fact that for any $j=1, \ldots, k$,

$$
\sum_{n \in \mathbb{Z}}\left|a_{n}\left(f_{j}\right)\right| n^{2}\left(\mathbb{1}_{n \geq 0}(b+\varepsilon)^{2 n}+\mathbb{1}_{n \leq 0}(a-\varepsilon)^{2 n}\right)<\infty,
$$

we deduce that for any $\delta>0$, there is $n_{0}>0$ fixed such that for all $N$ large enough,

$$
\sum_{j=1}^{k} \mathbb{E}\left|\mathbb{1}_{\mathcal{E}} \sum_{|n|>n_{0}} a_{n}\left(f_{j}\right) \operatorname{Tr}\left(\mathbf{A}^{n} \mathbf{M}_{j}\right)\right| \leq \delta
$$

for $\mathcal{E}=\mathcal{E}_{N}$ as in Lemma 3.2 .

Let us now suppose Theorem 2.4 to be proved in the particular case where there is $n_{0}$ such that for all $n$, we have

$$
|n|>n_{0} \Longrightarrow \forall j=1, \ldots, k, a_{n}\left(f_{j}\right)=0
$$

and let us prove it in the general case. Let $X_{N}$ denote the random vector of (3). We want to prove that as $N \rightarrow \infty$, the distribution of $X_{N}$ tends to the one of $\mathcal{G}:=\left(\mathcal{G}\left(f_{1}\right), \ldots, \mathcal{G}\left(f_{k}\right)\right)$, i.e. that for any function $F: \mathbb{C}^{k} \rightarrow \mathbb{C}$ which is 1 Lipschitz and bounded by 1 , we have

$$
\mathbb{E} F\left(X_{N}\right) \underset{N \rightarrow \infty}{\longrightarrow} \mathbb{E} F(\mathcal{G})
$$


To do so, we first set

$$
X_{N, n_{0}}:=\left(\sum_{|n|<n_{0}} a_{n}\left(f_{j}\right) \operatorname{Tr}\left(\mathbf{A}^{n} \mathbf{M}_{j}\right)-\operatorname{Tr}\left(\mathbf{M}_{j}\right) a_{0}\left(f_{j}\right)\right)_{j=1}^{k}
$$

By hypothesis, for any fixed $n_{0}, X_{N, n_{0}}$ converges in distribution to a centered complex Gaussian vector $\mathcal{G}_{n_{0}}:=\left(\mathcal{G}_{n_{0}}\left(f_{1}\right), \ldots, \mathcal{G}_{n_{0}}\left(f_{k}\right)\right)$ such that

$$
\begin{aligned}
\mathbb{E}\left[\mathcal{G}_{n_{0}}\left(f_{i}\right) \mathcal{G}_{n_{0}}\left(f_{j}\right)\right] & =\mathbb{E}\left[\mathcal{G}\left(f_{i}\right) \mathcal{G}\left(f_{j}\right)\right]+\eta_{i j}^{n_{0}} \\
\mathbb{E}\left[\mathcal{G}_{n_{0}}\left(f_{i}\right) \overline{\mathcal{G}_{n_{0}}\left(f_{j}\right)}\right] & =\mathbb{E}\left[\mathcal{G}\left(f_{i}\right) \overline{\mathcal{G}\left(f_{j}\right)}\right]+\delta_{i j}^{n_{0}},
\end{aligned}
$$

where $\lim _{n_{0} \rightarrow \infty} \sum_{1 \leq i, j \leq k}\left|\eta_{i j}^{n_{0}}\right|+\left|\delta_{i j}^{n_{0}}\right|=0$. Therefore,

$$
\begin{aligned}
& \left|\mathbb{E}\left[F\left(X_{N}\right)-F(\mathcal{G})\right]\right| \\
\leq & \left|\mathbb{E}\left[F\left(X_{N}\right)-F\left(X_{N, n_{0}}\right)\right]\right|+\left|\mathbb{E}\left[F\left(X_{N, n_{0}}\right)-F\left(\mathcal{G}_{n_{0}}\right)\right]\right|+\left|\mathbb{E}\left[F\left(\mathcal{G}_{n_{0}}\right)-F(\mathcal{G})\right]\right| \\
\leq & 2 \mathbb{P}\left(\mathcal{E}^{c}\right)+\mathbb{E}\left[\mathbb{1}_{\mathcal{E}}\left\|X_{N}-X_{N, n_{0}}\right\|_{2}^{2}\right]+\left|\mathbb{E}\left[F\left(X_{N, n_{0}}\right)-F\left(\mathcal{G}_{n_{0}}\right)\right]\right|+\mathbb{E}\left[\left\|\mathcal{G}_{n_{0}}-\mathcal{G}\right\|_{2}^{2}\right]
\end{aligned}
$$

which can be as small as we want by (7) and the fact that $X_{N, n_{0}} \stackrel{(\mathrm{d})}{\longrightarrow} \mathcal{G}_{n_{0}}$ if $\mathcal{G}_{n_{0}}$ and $\mathcal{G}$ are coupled the right way.

3.3. Proof of Theorem 2.4 when the $f_{j}$ 's are polynomial in $z$ and $z^{-1}$. We suppose here that there is $n_{0}>0$ such that for all $n>n_{0}$ and all $1 \leq j \leq k, a_{n}\left(f_{j}\right)=0$. Without any loss of generality, we also assume that for all $j, a_{0}\left(f_{j}\right)=0$. In this case, any linear combination of the $\operatorname{Tr} f_{j}(\mathbf{A}) \mathbf{M}_{j}$ 's can be written

$$
G_{N}:=\sum_{j=1}^{k} \nu_{j} \operatorname{Tr} f_{j}(\mathbf{A}) \mathbf{M}_{j}=\sum_{|n| \leq n_{0}} \operatorname{Tr} \mathbf{A}^{n} \mathbf{N}_{n}
$$

where $\mathbf{N}_{n}:=\sum_{j=1}^{k} \nu_{j} a_{n}\left(f_{j}\right) \mathbf{M}_{j}$. Written this way, we notice that to prove that the limit distribution of $G_{N}$ is Gaussian, we simply have to prove that the random vector

$$
\left(\operatorname{Tr} \mathbf{A}^{n} \mathbf{N}_{n}\right)_{-n_{0} \leq n \leq n_{0}}
$$

converges in distribution to a Gaussian vector. We will prove it by computing the limit of the joint moments.

Before going any further, recall that by the preliminary randomization of the $\mathbf{N}_{j}$ 's from section 3.1, we suppose that $\mathbf{A}=\mathbf{U T}$ (instead of $\mathbf{A}=\mathbf{U T V}$ ) and that there is a Haardistributed random unitary matrix $\mathbf{V}$, independent of $\mathbf{U}$, such that for each $j, \mathbf{N}_{j}=$ $\mathbf{V} \widetilde{\mathbf{N}}_{j} \mathbf{V}^{*}$, with $\tilde{\mathbf{N}}_{j}$ a deterministic matrix.

We shall proceed in three steps: 
a) First, we prove the asymptotic independence of the random vectors

$$
\left(\operatorname{Tr} \mathbf{A}^{n} \mathbf{N}_{n}, \operatorname{Tr} \mathbf{A}^{-n} \mathbf{N}_{-n}\right)_{n \geq 1}
$$

by the factorization of the joint moments. More precisely, we prove, thanks to Corollary A.4, that for any $\left(p_{n}\right)_{n=1}^{n_{0}},\left(q_{n}\right)_{n=1}^{n_{0}},\left(r_{n}\right)_{n=1}^{n_{0}},\left(s_{n}\right)_{n=1}^{n_{0}}$,

$$
\begin{aligned}
& \mathbb{E}\left[\prod_{1 \leq n \leq n_{0}}\left(\operatorname{Tr} \mathbf{A}^{n} \mathbf{N}_{n}\right)^{p_{n}} \overline{\left(\operatorname{Tr} \mathbf{A}^{n} \mathbf{N}_{n}\right)^{q_{n}}}\left(\operatorname{Tr} \mathbf{A}^{-n} \mathbf{N}_{-n}\right)^{r_{n}} \overline{\left(\operatorname{Tr} \mathbf{A}^{-n} \mathbf{N}_{-n}\right)^{s_{n}}}\right] \\
= & \prod_{1 \leq n \leq n_{0}} \mathbb{E}\left[\left(\operatorname{Tr} \mathbf{A}^{n} \mathbf{N}_{n}\right)^{p_{n}} \overline{\left(\operatorname{Tr} \mathbf{A}^{n} \mathbf{N}_{n}\right)^{q_{n}}}\left(\operatorname{Tr} \mathbf{A}^{-n} \mathbf{N}_{-n}\right)^{r_{n}} \overline{\left(\operatorname{Tr} \mathbf{A}^{-n} \mathbf{N}_{-n}\right)^{s_{n}}}\right]+o(1)
\end{aligned}
$$

b) Then, we prove for any fixed $n$, the random complex vector

$$
\left(\operatorname{Tr} \mathbf{A}^{n} \mathbf{N}_{n}, \operatorname{Tr} \mathbf{A}^{-n} \mathbf{N}_{-n}\right)
$$

converges in distribution to a centered complex Gaussian vector thanks to the criterion provided by the Lemma A.7. This criterion consists in proving that the joint moments, at the large $N$ limit, satisfy the same induction relation as the moments of a Gaussian distribution.

c) It will follow from $\mathbf{a}$ ) and $\mathbf{b}$ ) that when all $f_{j}$ 's are polynomials in $z$ and $z^{-1}$, the random vector of (3) converges in distribution to a centered Gaussian vector. To conclude the proof, the last step will be to prove that the limit covariance is the one given in Theorem 2.4.

In the proofs of $\mathbf{a}$ ) and $\mathbf{b}$ ), we shall need to compute expectations with respect to the randomness of the Haar-distributed matrix $\mathbf{U}$. More precisely, we shall need to compute sums of expectations with respect of $\mathbf{U}$ resulting from the expansion of products of traces involving powers of $\mathbf{A}$ (such as $\operatorname{Tr} \mathbf{A}^{n} \mathbf{N}_{n}$ ). To do so, we will use the Weingarten calculus (see Proposition A.1) and shall always proceed in the following way: first, we use (28) to state that all the terms of the sum are null except those for which the left (resp. right) indices involved in $u$ are obtained by permuting the left (resp. right) ones involved in $\bar{u}$. Then, we claim, by Remark A.2, that among the remaining terms, we can neglect all those whose indices are not pairwise distinct. At last, once all the remaining terms are, up to multiplicative constant, equal to $\mathrm{Wg}(\sigma)$ for some permutation $\sigma$, we neglect all those for which $\sigma \neq i d$ (see Remark A.3) and the summation finally gets easy to compute. We introduce here a notation that we shall use several times :

$$
\mathrm{I}_{n}^{\neq}:=\left\{\left(i_{1}, \ldots, i_{n}\right) \in\{1, \ldots, N\}^{n} ; i_{1}, \ldots, i_{n} \text { are pairwise distinct }\right\}
$$

(this set implicitly depends on $N$ ).

3.4. Proof of $\mathbf{b})$. In this part, as $n$ is fixed, we shall denote $\mathbf{N}_{n}\left(\operatorname{resp} . \mathbf{N}_{-n}\right)$ by $\mathbf{M}=\left[M_{i j}\right]$ (resp. $\mathbf{K}=\left[K_{i j}\right]$ ). For any non-negative integers $p, q, r, s$, wet set

$$
m_{p, q, r, s}:=\mathbb{E}\left(\operatorname{Tr} \mathbf{A}^{n} \mathbf{M}\right)^{p}{\overline{\left(\operatorname{Tr} \mathbf{A}^{n} \mathbf{M}\right)}}^{q}\left(\operatorname{Tr} \mathbf{A}^{-n} \mathbf{K}\right)^{r}{\overline{\left(\operatorname{Tr} \mathbf{A}^{-n} \mathbf{K}\right)}}^{s}
$$


and our goal is to show that, as $N$ goes to infinity, the numbers $m_{p, q, r, s}$ have limits satisfying conditions (34), (35), (37), (38) and (39) of the Lemma A.7. Note that (34) and (37) follow from the fact the the Haar measure on the unitary group is invariant by multiplication by any $\mathrm{e}^{\mathrm{i} \theta}, \theta \in \mathbb{R}$. We shall use the following notations

$$
\begin{aligned}
\lim _{N \rightarrow \infty} \frac{1}{N} \operatorname{Tr}\left(\mathbf{M M}^{*}\right) & =: \alpha_{\mathbf{M}} ; \lim _{N \rightarrow \infty} \frac{1}{N} \operatorname{Tr}\left(\mathbf{K K}^{*}\right)=: \alpha_{\mathbf{K}} ; \lim _{N \rightarrow \infty} \frac{1}{N} \operatorname{Tr}(\mathbf{M K})=: \beta_{\mathbf{M}, \mathbf{K}} \\
\lim _{N \rightarrow \infty} \frac{1}{N} \operatorname{Tr} \mathbf{M} & =: \tau_{\mathbf{M}} ; \lim _{N \rightarrow \infty} \frac{1}{N} \operatorname{Tr} \mathbf{K}=: \tau_{\mathbf{K}} .
\end{aligned}
$$

3.4.1. $\operatorname{Tr} \mathbf{A}^{n} \mathbf{M}$ and $\operatorname{Tr} \mathbf{A}^{-n} \mathbf{K}$ are asymptotically two circular Gaussian complex variables satisfying conditions (35) and (39). We simply have to show that for any integer $p \geq 1$

$$
\begin{aligned}
\mathbb{E}\left|\operatorname{Tr} \mathbf{A}^{n} \mathbf{M}\right|^{2 p} & =p !\left(b^{2 n}\left((n-1)\left|\tau_{\mathbf{M}}\right|^{2}+\alpha_{\mathbf{M}}\right)\right)^{p}+o(1), \\
\mathbb{E}\left|\operatorname{Tr} \mathbf{A}^{-n} \mathbf{K}\right|^{2 p} & =p !\left(a^{-2 n}\left((n-1)\left|\tau_{\mathbf{K}}\right|^{2}+\alpha_{\mathbf{K}}\right)\right)^{p}+o(1), \\
\mathbb{E}\left(\operatorname{Tr} \mathbf{A}^{n} \mathbf{M} \operatorname{Tr} \mathbf{A}^{-n} \mathbf{K}\right)^{p} & =p !\left((n-1) \tau_{\mathbf{M}} \tau_{\mathbf{K}}+\beta_{\mathbf{M}, \mathbf{K}}\right)^{p}+o(1) .
\end{aligned}
$$

We shall prove it by induction on $p$. So first, we show the previous relation for $p=1$. Recall that we assume that $\mathbf{M}=\mathbf{V} \widetilde{\mathbf{M}} \mathbf{V}^{*}$ and $\mathbf{K}=\mathbf{V} \widetilde{\mathbf{K}} \mathbf{V}^{*}$, where $\widetilde{\mathbf{M}}$ and $\widetilde{\mathbf{K}}$ are deterministic, so that, using the Lemma A.6, we have (denoting again by $\mathbb{E}_{\mathbf{V}}$ the expectation with respect to the randomness of $\mathbf{V}$ ),

$$
\begin{aligned}
\mathbb{E}_{\mathbf{V}}\left|\operatorname{Tr} \mathbf{A}^{n} \mathbf{V} \widetilde{\mathbf{M}} \mathbf{V}^{*}\right|^{2} & =\frac{1}{N} \operatorname{Tr} \mathbf{A}^{n}\left(\mathbf{A}^{*}\right)^{n}\left(\frac{1}{N} \operatorname{Tr} \widetilde{\mathbf{M}} \widetilde{\mathbf{M}}^{*}-\left|\frac{1}{N} \operatorname{Tr} \widetilde{\mathbf{M}}\right|^{2}\right) \\
& +\left|\operatorname{Tr} \mathbf{A}^{n}\right|^{2}\left|\frac{1}{N} \operatorname{Tr} \widetilde{\mathbf{M}}\right|^{2}+O\left(\frac{1}{N}\right) \\
\mathbb{E}_{\mathbf{V}}\left|\operatorname{Tr} \mathbf{A}^{-n} \mathbf{V} \widetilde{\mathbf{K}} \mathbf{V}^{*}\right|^{2} & =\frac{1}{N} \operatorname{Tr} \mathbf{A}^{-n}\left(\mathbf{A}^{*}\right)^{-n}\left(\frac{1}{N} \operatorname{Tr}(\widetilde{\mathbf{K}} \widetilde{\mathbf{K}})-\left|\frac{1}{N} \operatorname{Tr} \widetilde{\mathbf{K}}\right|^{2}\right) \\
& +\left|\operatorname{Tr} \mathbf{A}^{-n}\right|^{2}\left|\frac{1}{N} \operatorname{Tr} \widetilde{\mathbf{K}}\right|^{2}+O\left(\frac{1}{N}\right)
\end{aligned}
$$

$\mathbb{E}_{\mathbf{V}} \operatorname{Tr} \mathbf{A}^{n} \mathbf{V} \widetilde{\mathbf{M}} \mathbf{V} \operatorname{Tr} \mathbf{A}^{-n} \mathbf{V} \widetilde{\mathbf{K}} \mathbf{V}^{*}=\frac{1}{N} \operatorname{Tr} \widetilde{\mathbf{M}} \widetilde{\mathbf{K}}-\frac{1}{N} \operatorname{Tr} \widetilde{\mathbf{M}} \frac{1}{N} \operatorname{Tr} \widetilde{\mathbf{K}}$

$$
+\operatorname{Tr} \mathbf{A}^{n} \operatorname{Tr} \mathbf{A}^{-n} \frac{1}{N} \operatorname{Tr} \widetilde{\mathbf{M}} \frac{1}{N} \operatorname{Tr} \widetilde{\mathbf{K}}+O\left(\frac{1}{N}\right)
$$

This is asymptotically determined by the limits of $\mathbb{E}\left|\operatorname{Tr} \mathbf{A}^{n}\right|^{2}, \mathbb{E}\left|\operatorname{Tr} \mathbf{A}^{-n}\right|^{2}, \mathbb{E} \operatorname{Tr} \mathbf{A}^{n} \operatorname{Tr} \mathbf{A}^{-n}$, $N^{-1} \mathbb{E} \operatorname{Tr} \mathbf{A}^{n}\left(\mathbf{A}^{*}\right)^{n}$ and $N^{-1} \mathbb{E} \operatorname{Tr} \mathbf{A}^{-n}\left(\mathbf{A}^{*}\right)^{-n}$. First, we compute $\mathbb{E}\left|\operatorname{Tr} \mathbf{A}^{n}\right|^{2}$ for $n \geq 1$. We write

$$
\mathbb{E}\left|\operatorname{Tr} \mathbf{A}^{n}\right|^{2}=\sum_{\substack{1 \leq i_{1}, \ldots, i_{n} \leq N \\ 1 \leq j_{1}, \ldots, j_{n} \leq N}} \mathbb{E}\left[u_{i_{1} i_{2}} \cdots u_{i_{n} i_{1}} \overline{u_{j_{1} j_{2}}} \cdots \overline{u_{j_{n} j_{1}}}\right] s_{i_{1}} s_{j_{1}} \cdots s_{i_{n}} s_{j_{n}}
$$


From (28), we have a condition on the $i_{k}$ 's and the $j_{k}$ 's for a non-vanishing expectation, which is the multiset ${ }^{1}$ equality

$$
\left\{i_{1}, \ldots, i_{n}\right\}_{m}=\left\{j_{1}, \ldots, j_{n}\right\}_{m}
$$

The first consequence of (13) is that the sum is in fact over $O\left(N^{n}\right)$ terms which all are at most $O\left(N^{-n}\right)$, which means that any sub-summation over $o\left(N^{n}\right)$ terms might be neglected. So from now on, we shall only sum over the $n$-tuples $\left(i_{1}, \ldots, i_{n}\right) \in \mathrm{I}_{n}^{\neq}$(recall notation (8)). Then (12) becomes

$$
\mathbb{E}\left|\operatorname{Tr} \mathbf{A}^{n}\right|^{2}=\sum_{\left(i_{1}, \ldots, i_{n}\right) \in \mathrm{I}_{n}^{f}} s_{i_{1}}^{2} \cdots s_{i_{n}}^{2} \sum_{\sigma \in S_{n}} \mathbb{E}\left[u_{i_{1} i_{2}} \cdots u_{i_{n} i_{1}} \overline{u_{i_{\sigma(1)} i_{\sigma(2)}}} \cdots \overline{u_{i_{\sigma(n)} i_{\sigma(1)}}}\right]+o(1)
$$

Let $c \in S_{n}$ be the cycle $(12 \cdots n)$. From (28) (see Remark A.2), as long as the $i_{k}$ 's are pairwise distinct, one can write

$$
\mathbb{E}\left[u_{i_{1} i_{2}} \cdots u_{i_{n} i_{1}} \overline{u_{i_{\sigma(1)} i_{\sigma(2)}}} \cdots \overline{u_{i_{\sigma(n)} i_{\sigma(1)}}}\right]=\mathrm{Wg}\left(\sigma c^{-1} \sigma^{-1} c\right)
$$

and from (29) and Remark A.3, we know that the non-negligible terms are the ones such that $\sigma c^{-1} \sigma^{-1} c=i d$, i.e. $\sigma c=c \sigma$, which means that $\sigma$ must be a power of $c$ and so, only $n$ permutations $\sigma$ contribute to the non negligible terms. At last, as $\operatorname{Moeb}(i d)=1$, we have

$$
\begin{aligned}
\mathbb{E}\left|\operatorname{Tr} \mathbf{A}^{n}\right|^{2} & =\sum_{\left(i_{1}, \ldots, i_{n}\right) \in \mathrm{I}_{n}^{\neq}} s_{i_{1}}^{2} \cdots s_{i_{n}}^{2} \times n N^{-n}(1+o(1))+o(1) \\
& =n\left(\frac{1}{N} \sum_{i=1}^{N} s_{i}^{2}\right)^{n}+o(1)=n b^{2 n}+o(1) .
\end{aligned}
$$

In the same way, one can get

$$
\begin{aligned}
\mathbb{E}\left|\operatorname{Tr} \mathbf{A}^{-n}\right|^{2} & =n a^{-2 n}+o(1), \\
\mathbb{E} \operatorname{Tr} \mathbf{A}^{n} \operatorname{Tr} \mathbf{A}^{-n} & =n+o(1) .
\end{aligned}
$$

Let us now consider $N^{-1} \mathbb{E} \operatorname{Tr} \mathbf{A}^{n}\left(\mathbf{A}^{*}\right)^{n}$ for $n \geq 1$. We have

$$
\frac{1}{N} \mathbb{E} \operatorname{Tr} \mathbf{A}^{n}\left(\mathbf{A}^{*}\right)^{n}=N_{\substack{1 \leq i_{0}, i_{1}, \ldots, i_{n} \leq N \\ 1 \leq j_{0}, j_{1}, \ldots, j_{n} \leq N \\ i_{0}=j_{0}, i_{n}=j_{n}}}^{-1} \mathbb{E}\left[u_{i_{0} i_{1}} \cdots u_{i_{n-1} i_{n}} \overline{u_{j_{0} j_{1}}} \cdots \overline{u_{j_{n-1} j_{n}}}\right] s_{i_{1}} s_{j_{1}} \cdots s_{i_{n}} s_{j_{n}}
$$

As previously, we know that by (28), that for the expectation to be non zero, we must have the multiset equality

$$
\left\{i_{0}, \ldots, i_{n}\right\}_{m}=\left\{j_{0}, \ldots, j_{n}\right\}_{m}
$$

The first consequence of $(15)$ is that the sum is in fact over $O\left(N^{n+1}\right)$ terms which are all at most $O\left(N^{-n-1}\right)$, so that any sub-summation over $o\left(N^{n+1}\right)$ terms might be neglected. As

\footnotetext{
${ }^{1}$ We use the index $m$ in $\{\cdot\}_{m}$ to denote a multiset, which means that $\left\{x_{1}, \ldots, x_{n}\right\}_{m}$ is the class of the $n$-tuple $\left(x_{1}, \ldots, x_{n}\right)$ under the action of the symmetric group $S_{n}$.
} 
previously, we shall sum over the pairwise distinct indices $\mathrm{I}_{n+1}^{\neq}$(see notation (8)). Hence (14) becomes

$$
N^{-1} \mathbb{E} \operatorname{Tr} \mathbf{A}^{n}\left(\mathbf{A}^{*}\right)^{n}=N^{-1} \sum_{\left(i_{0}, i_{1}, \ldots, i_{n}\right) \in \mathrm{I}_{n+1}^{\neq}} s_{i_{1}}^{2} \cdots s_{i_{n}}^{2} \sum_{\substack{\sigma \in S_{n+1} \\ \sigma(0)=0 \\ \sigma(n)=n}} \mathbb{E}\left[u_{i_{0} i_{1}} \cdots u_{i_{n-1} i_{n}} \overline{u_{i_{\sigma(0)} i_{\sigma(1)}}} \cdots \overline{u_{i_{\sigma(n-1)} i_{\sigma(n)}}}\right]
$$

Let $c \in S_{n+1}$ be the cycle $\left(\begin{array}{llll}0 & 1 & 2 & \cdots\end{array}\right)$. From (28) (see Remark A.2) one can write

$$
\mathbb{E}\left[u_{i_{0} i_{1}} \cdots u_{i_{n-1} i_{n}} \overline{u_{i_{\sigma(0)} i_{\sigma(1)}}} \cdots \overline{u_{i_{\sigma(n-1)} i_{\sigma(n)}}}\right]=\operatorname{Wg}\left(\sigma c^{-1} \sigma^{-1} c\right) .
$$

As previously, $\sigma$ must be a power of $c$ for the asymptotic contribution to be non-negligible. However, this time, we impose $\sigma(0)=0$ and $\sigma(n)=n$, so that the only possible choice is $\sigma=i d$ which means that only one term contributes this time. At last,

$$
\frac{1}{N} \mathbb{E} \operatorname{Tr} \mathbf{A}^{n}\left(\mathbf{A}^{*}\right)^{n}=b^{2 n}+o(1)
$$

The same way, one can get

$$
\frac{1}{N} \mathbb{E} \operatorname{Tr} \mathbf{A}^{-n}\left(\mathbf{A}^{*}\right)^{-n}=a^{-2 n}+o(1) .
$$

This concludes the first step of the induction.

In the second step, we have to prove the following induction relation: for any $p \geq 2$,

$$
\begin{aligned}
& \mathbb{E}\left|\operatorname{Tr} \mathbf{A}^{n} \mathbf{M}\right|^{2 p}=p \mathbb{E}\left|\operatorname{Tr} \mathbf{A}^{n} \mathbf{M}\right|^{2} \mathbb{E}\left|\operatorname{Tr} \mathbf{A}^{n} \mathbf{M}\right|^{2(p-1)}+o(1) \\
& \mathbb{E}\left|\operatorname{Tr} \mathbf{A}^{-n} \mathbf{K}\right|^{2 p}=p \mathbb{E}\left|\operatorname{Tr} \mathbf{A}^{-n} \mathbf{K}\right|^{2} \mathbb{E}\left|\operatorname{Tr} \mathbf{A}^{-n} \mathbf{K}\right|^{2(p-1)}+o(1) \\
& \mathbb{E}\left(\operatorname{Tr} \mathbf{A}^{n} \mathbf{M} \operatorname{Tr} \mathbf{A}^{-n} \mathbf{K}\right)^{p}=p \mathbb{E}\left[\operatorname{Tr} \mathbf{A}^{n} \mathbf{M} \operatorname{Tr} \mathbf{A}^{-n} \mathbf{K}\right] \mathbb{E}\left[\left(\operatorname{Tr} \mathbf{A}^{n} \mathbf{M} \operatorname{Tr} \mathbf{A}^{-n} \mathbf{K}\right)^{p-1}\right] \\
&+o(1)
\end{aligned}
$$

Let us first consider $\mathbb{E}\left|\operatorname{Tr} \mathbf{A}^{n} \mathbf{M}\right|^{2 p}$. We shall use the following notation

$$
\operatorname{Tr} \mathbf{A}^{n} \mathbf{M}=\sum_{i_{0}, i_{1}, \ldots, i_{n}} u_{i_{0} i_{1}} s_{i_{1}} \cdots u_{i_{n-1} i_{n}} s_{i_{n}} M_{i_{n} i_{0}}=: \sum_{\mathbf{i}} u_{\mathbf{i}} s_{\mathbf{i}} M_{i_{n} i_{0}},
$$

where the bold letter $\mathbf{i}$ denotes the $(n+1)$-tuple $\left(i_{0}, \ldots, i_{n}\right)$ and where we set

$$
u_{\mathbf{i}}:=u_{i_{0} i_{1}} \cdots u_{i_{n-1} i_{n}} \quad ; \quad s_{\mathbf{i}}:=s_{i_{1}} \cdots s_{i_{n}} .
$$

Hence,

$$
\mathbb{E}\left|\operatorname{Tr} \mathbf{A}^{n} \mathbf{M}\right|^{2 p}=\sum_{\substack{\mathbf{i}^{1}, \ldots, \mathbf{i}^{p} \\ \mathbf{j}^{1}, \ldots, \mathbf{j}^{p}}} \mathbb{E}\left[u_{\mathbf{i}^{1}} \cdots u_{\mathbf{i}^{p}} \overline{u_{\mathbf{j}^{1}}} \cdots \overline{u_{\mathbf{j}^{p}}}\right] s_{\mathbf{i}^{1}} M_{i_{n}^{1} i_{0}^{1}} s_{\mathbf{j}^{1}} \overline{M_{j_{n}^{1} j_{0}^{1}}} \cdots s_{\mathbf{i}^{p}} M_{i_{n}^{p} i_{0}^{p}} s_{\mathbf{j}^{p}} \overline{M_{j_{n}^{p} j_{0}^{p}}}
$$

As usual, we know we can sum over the $\mathbf{i}^{k}$ 's satisfying that $\left(\mathbf{i}^{1}, \ldots, \mathbf{i}^{p}\right)$ (the $p(n+1)$-tuple obtained by concatenation of the $\mathbf{i}$ 's) has pairwise distinct entries and such that we have the set equality:

$$
\left\{i_{\mu}^{\lambda}, 1 \leq \lambda \leq p, 0 \leq \mu \leq n\right\}=\left\{j_{\mu}^{\lambda}, 1 \leq \lambda \leq p, 0 \leq \mu \leq n\right\}
$$


Then, in order to have $\mathrm{Wg}(i d)$, we must match each of the $(n+1)$-tuples $\mathbf{i}^{1}, \ldots, \mathbf{i}^{p}$ with one of the $(n+1)$-tuples $\mathbf{j}^{1}, \ldots, \mathbf{j}^{p}$, i.e. that for all $1 \leq \lambda \leq p$, there is a $1 \leq \lambda^{\prime} \leq p$ such that we have the set equality

$$
\left\{\mathbf{i}^{\lambda}\right\}=:\left\{i_{0}^{\lambda}, i_{1}^{\lambda}, \ldots, i_{n}^{\lambda}\right\}=\left\{j_{0}^{\lambda^{\prime}}, j_{1}^{\lambda^{\prime}}, \ldots, j_{n}^{\lambda^{\prime}}\right\}:=\left\{\mathbf{j}^{\lambda^{\prime}}\right\}
$$

We rewrite (20) by summing according the possible choice to match $\left\{\mathbf{i}^{1}\right\}=\left\{i_{0}^{1}, i_{1}^{1}, \ldots, i_{n}^{1}\right\}$

$$
\mathbb{E}\left|\operatorname{Tr} \mathbf{A}^{n} \mathbf{M}\right|^{2 p}=\sum_{\substack{\lambda=1 \\\left(\mathbf{i}^{1}, \ldots, \mathbf{i}^{p}\right) \in \mathrm{I}_{p(n+1)}^{\neq} \\\left(\mathbf{j}^{1}, \ldots, \mathbf{j}^{p}\right) \in \mathrm{I}_{p(n+1)}^{\neq} \\ \mathbf{i}^{1} \leftrightarrow \mathbf{j}^{\lambda}}} \mathbb{E}\left[u_{\mathbf{i}^{1}} \cdots u_{\mathbf{i}^{p}} \overline{u_{\mathbf{j}^{1}}} \cdots \overline{u_{\mathbf{j}^{p}}}\right] s_{\mathbf{i}^{1}} M_{i_{n}^{1} i_{0}^{1}} s_{\mathbf{j}^{1}} \overline{M_{j_{n}^{1} j_{0}^{1}}} \cdots s_{\mathbf{i}^{p}} M_{i_{n}^{p} p_{0}^{p}} s_{\mathbf{j}^{p}} \overline{M_{j_{n}^{p} j_{0}^{p}}^{p}}+o(1),
$$

where $\mathbf{i}^{1} \leftrightarrow \mathbf{j}^{\lambda}$ stands for the set equality $\left\{i_{0}^{1}, i_{1}^{1}, \ldots, i_{n}^{1}\right\}=\left\{j_{0}^{\lambda}, j_{1}^{\lambda}, \ldots, j_{n}^{\lambda}\right\}$. Then, we know that the set of indices $\left\{i_{0}^{1}, i_{1}^{1}, \ldots, i_{n}^{1}\right\}$ is disjoint from the others, so that by Corollary A.4,

$$
\mathbb{E}\left[u_{\mathbf{i}^{1}} \cdots u_{\mathbf{i}^{p}} \overline{u_{\mathbf{j}^{1}}} \cdots \overline{u_{\mathbf{j}^{p}}}\right]=\mathbb{E}\left[u_{\mathbf{i}^{1}} \overline{u_{\mathbf{j}^{\lambda}}}\right] \mathbb{E}\left[u_{\mathbf{i}^{2}} \cdots u_{\mathbf{i}^{p}} \overline{u_{\mathbf{j}^{1}}} \cdots \overline{u_{\mathbf{j}^{\lambda-1}} u_{\mathbf{j}^{\lambda+1}}} \cdots \overline{u_{\mathbf{j}^{p}}}\right]
$$

and up to a proper relabeling of the indices, all the choices lead to the same value of the expectation, so that

$$
\begin{aligned}
& \mathbb{E}\left|\operatorname{Tr} \mathbf{A}^{n} \mathbf{M}\right|^{2 p} \\
& =p \sum_{\substack{\mathbf{i}^{1} \in \mathrm{I}_{n+1}^{\neq} \\
\mathbf{j}^{1} \in \mathrm{I}_{n+1}^{\neq}}} \mathbb{E}\left[u_{\mathbf{i}^{1}} \overline{u_{\mathbf{j}^{1}}}\right] s_{\mathbf{i}^{1}} M_{i_{n}^{1}, i_{0}^{1}} s_{\mathbf{j}^{1}} \overline{M_{j_{n}^{1}, j_{0}^{1}}} \sum_{\substack{\left(\mathbf{i}^{2}, \ldots, \mathbf{i}^{p}\right) \in \mathrm{I}_{(p-1)(n+1)}^{\neq} \\
\left(\mathbf{j}^{2}, \ldots, \mathbf{j}^{p}\right) \in \mathrm{I}_{(p-1)(n+1)}^{\neq}}} \mathbb{E}\left[u_{\mathbf{i}^{2}} \cdots \overline{u_{\mathbf{j}^{p}}}\right] s_{\mathbf{i}^{2}} M_{i_{n}^{2} i_{0}^{2}} s_{\mathbf{j}^{2}} \overline{M_{j_{n}^{2} j_{0}^{2}}} \cdots s_{\mathbf{i}^{p}} M_{i_{n}^{p} i_{0}^{p}} s_{\mathbf{j}^{p}} \overline{M_{j_{n}^{p} j_{0}^{p}}}+o(1) \\
& =p \mathbb{E}\left[\left|\operatorname{Tr}\left(\mathbf{A}^{n} \mathbf{M}\right)\right|^{2}\right] \mathbb{E}\left[\left|\operatorname{Tr}\left(\mathbf{A}^{n} \mathbf{M}\right)\right|^{2(p-1)}\right]+o(1) \text {. }
\end{aligned}
$$

This proves (16). In the same way, we prove (17) and (18), and thus conclude the proof of the induction.

Remark 3.3. In the last computation, we split the expectation and so we separated the summation implying that

$$
\mathrm{I}_{p(n+1)}^{\neq}=\mathrm{I}_{n+1}^{\neq} \times \mathrm{I}_{(p-1)(n+1)}^{\neq}
$$

which is obviously inaccurate. Nevertheless, we easily see that

$$
\operatorname{Card} \mathrm{I}_{p(n+1)}^{\neq}=\operatorname{Card}\left(\mathrm{I}_{n+1}^{\neq} \times \mathrm{I}_{(p-1)(n+1)}^{\neq}\right)(1+o(1))
$$

which means that this inaccuracy is actually contained in the $o(1)$.

To conclude the proof of $\mathbf{b}$ ), we have to prove that $\operatorname{Tr} \mathbf{A}^{n} \mathbf{M}$ and $\operatorname{Tr} \mathbf{A}^{-n} \mathbf{K}$ satisfy Condition (38) at the large $N$ limit. 
FLUCTUATIONS FOR ANALYTIC TEST FUNCTIONS IN THE SINGLE RING THEOREM

3.4.2. $\operatorname{Tr} \mathbf{A}^{n} \mathbf{M}$ and $\operatorname{Tr} \mathbf{A}^{-n} \mathbf{K}$ satisfy Condition (38) at the large $N$ limit. We apply the same idea as previously, but for a slightly more complicated expectation. Let $p, q, r, s$ be positive integers and such that $p-q=r-s$. We denote joint moments by $m_{p, q, r, s}$ :

$$
m_{p, q, r, s}:=\mathbb{E}\left(\operatorname{Tr} \mathbf{A}^{n} \mathbf{M}\right)^{p}{\overline{\left(\operatorname{Tr} \mathbf{A}^{n} \mathbf{M}\right)^{q}}}^{q}\left(\operatorname{Tr} \mathbf{A}^{-n} \mathbf{K}\right)^{r}{\overline{\left(\operatorname{Tr} \mathbf{A}^{-n} \mathbf{K}\right)}}^{s},
$$

and as

$$
\begin{aligned}
\operatorname{Tr} \mathbf{A}^{n} \mathbf{M} & =\sum_{i_{0}, i_{1}, \ldots, i_{n}} u_{i_{0} i_{1}} s_{i_{1}} \cdots u_{i_{n-1} i_{n}} s_{i_{n}} M_{i_{n} i_{0}}=\sum_{\mathbf{i}} u_{\mathbf{i}} s_{\mathbf{i}} M_{i_{n} i_{0}} \\
\operatorname{Tr} \mathbf{A}^{-n} \mathbf{K} & =\sum_{i_{n}, i_{n-1}, \ldots, i_{0}} \bar{u}_{i_{n-1} i_{n}} s_{i_{n}}^{-1} \cdots \bar{u}_{i_{0} i_{1}} s_{i_{1}} K_{i_{0} i_{n}}=\sum_{\mathbf{i}} \bar{u}_{\mathbf{i}} s_{\mathbf{i}}^{-1} K_{i_{0} i_{n}},
\end{aligned}
$$

we rewrite (22) as follow

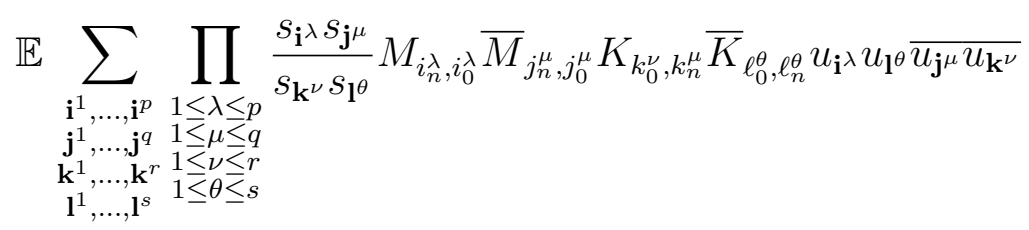

(recall that the $s_{\mathbf{i}}=s_{i_{1}} \cdots s_{i_{n}}$ for $\mathbf{i}=\left(i_{0}, \ldots, i_{n}\right)$ ). As previously, we deduce from Proposition A.1 that for the non vanishing expectations, we must have the following multiset equality

$$
\begin{aligned}
& \left\{i_{\mu}^{\lambda}, 1 \leq \lambda \leq p, 0 \leq \mu \leq n\right\}_{m} \cup\left\{\ell_{\mu}^{\lambda}, 1 \leq \lambda \leq s, 0 \leq \mu \leq n\right\}_{m} \\
= & \left\{j_{\mu}^{\lambda}, 1 \leq \lambda \leq q, 0 \leq \mu \leq n\right\}_{m} \cup\left\{k_{\mu}^{\lambda}, 1 \leq \lambda \leq r, 0 \leq \mu \leq n\right\}_{m},
\end{aligned}
$$

from which we deduce that we can restrict the summation to the tuples such that

$$
\left(\mathbf{i}^{1}, \ldots, \mathbf{i}^{p}, \mathbf{l}^{1}, \ldots \mathbf{l}^{s}\right) \in \mathrm{I}_{(p+s)(n+1)}^{\neq}
$$

and that, for the non negligible terms (i.e. those which lead to $\mathrm{Wg}(i d)$ ), we must match each of the $(n+1)$-tuples involved in $u$ (the i's and the l's) with one of those involved in $\bar{u}$ (the $\mathbf{j}$ 's and the $\mathbf{k}$ 's). For example, we sum according to the choice the "partner" of $\mathbf{i}^{1}$.

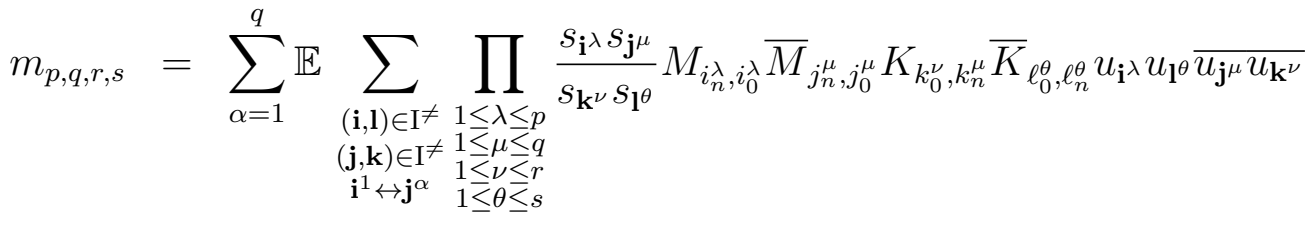

$$
\begin{aligned}
& +\sum_{\beta=1}^{r} \mathbb{E} \sum_{\substack{(\mathbf{i}, \mathbf{l}) \in \mathrm{I}^{\neq} \neq \\
\left(\mathbf{j}, \mathbf{k} \in \mathrm{I}^{\neq} \\
\mathbf{i}^{1} \leftrightarrow \mathbf{k}^{\beta}\right.}} \prod_{\substack{1 \leq \lambda \leq p \leq r \\
1 \leq \nu \leq r \\
1 \leq \theta \leq s}} \frac{s_{\mathbf{i}^{\lambda}} s_{\mathbf{j}^{\mu}}}{s_{\mathbf{k}^{\nu}} s_{\mathbf{l}^{\theta}}} M_{i_{n}^{\lambda}, i_{0}^{\lambda}} \bar{M}_{j_{n}^{\mu}, j_{0}^{\mu}} K_{k_{0}^{\nu}, k_{n}^{\mu}} \bar{K}_{\ell_{0}^{\theta}, \ell_{n}^{\theta}} u_{\mathbf{i}^{\lambda}} u_{\mathbf{l}^{\theta}} \overline{u_{\mathbf{j}^{\mu}} u_{\mathbf{k}^{\nu}}}+o(1) .
\end{aligned}
$$

where, to simplify, $(\mathbf{i}, \mathbf{l})$ stands for the $(p+s)(n+1)$-tuple obtained by the concatenation of the $\mathbf{i}^{\lambda}$ 's and the $\mathbf{I}^{\mu}$ s, and $\mathrm{I}^{\neq}$implicitly means $\mathrm{I}_{(p+s)(n+1)}^{\neq}$. As previously, we use the 
Corollary A.4 to split the expectations. Hence, one easily gets

$$
m_{p, q, r, s}=q \mathbb{E}\left[\left|\operatorname{Tr} \mathbf{A}^{n} \mathbf{M}\right|^{2}\right] m_{p-1, q-1, r, s}+r \mathbb{E}\left[\operatorname{Tr} \mathbf{A}^{n} \mathbf{M} \operatorname{Tr} \mathbf{A}^{-n} \mathbf{K}\right] m_{p-1, q, r-1, s}+o(1) .
$$

To get the other relations, we just sum according to the choice of the partner of $\mathbf{j}^{1}$ (resp. $\mathbf{k}^{1}$ and $\mathbf{l}^{1}$ ).

3.5. Proof of a): asymptotic factorisation of joint moments. The proof relies mostly on Corollary A.4. We first expand the expectation

$$
\mathbb{E}\left[\prod_{1 \leq n \leq n_{0}}\left(\operatorname{Tr} \mathbf{A}^{n} \mathbf{N}_{n}\right)^{p_{n}} \overline{\left(\operatorname{Tr} \mathbf{A}^{n} \mathbf{N}_{n}\right)^{q_{n}}}\left(\operatorname{Tr} \mathbf{A}^{-n} \mathbf{N}_{-n}\right)^{r_{n}} \overline{\left(\operatorname{Tr} \mathbf{A}^{-n} \mathbf{N}_{-n}\right)^{s_{n}}}\right]
$$

Let $M_{i j}^{(n)}$ denote the $(i, j)$-th entry of $\mathbf{N}_{n}$ and recall that for $\mathbf{i}=\left(i_{0}, \ldots, i_{n}\right)$, we set

$$
u_{\mathbf{i}}:=u_{i_{0} i_{1}} \cdots u_{i_{n-1} i_{n}} \quad ; \quad s_{\mathbf{i}}:=s_{i_{1}} \cdots s_{i_{n}} .
$$

We get

$$
\begin{aligned}
\operatorname{Tr} \mathbf{A}^{n} \mathbf{N}_{n} & =\sum_{i_{0}, i_{1}, \ldots, i_{n}} u_{i_{0} i_{1}} s_{i_{1}} \cdots u_{i_{n-1} i_{n}} s_{i_{n}} M_{i_{n} i_{0}}^{(n)}=\sum_{\mathbf{i}} u_{\mathbf{i}} s_{\mathbf{i}} M_{i_{n} i_{0}}^{(n)} \\
\operatorname{Tr} \mathbf{A}^{-n} \mathbf{N}_{-n} & =\sum_{i_{n}, i_{n-1}, \ldots, i_{0}} \bar{u}_{i_{n_{1}} i_{n}} s_{i_{n}}^{-1} \cdots \bar{u}_{i_{0} i_{1}} s_{i_{1}} M_{i_{0} i_{n}}^{(-n)}=\sum_{\mathbf{i}} \bar{u}_{\mathbf{i}} s_{\mathbf{i}}^{-1} M_{i_{0} i_{n}}^{(-n)},
\end{aligned}
$$

so that

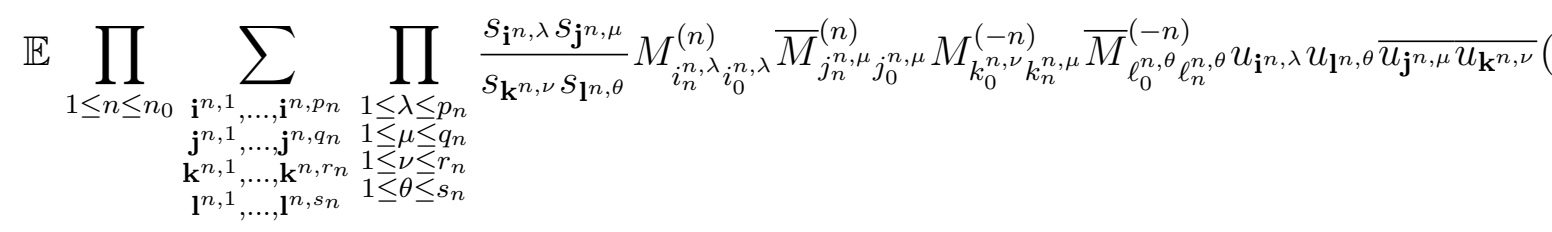

where we use bold letters such as $\mathbf{i}^{n, \lambda}$ to denote $(n+1)$-tuples $\left(i_{0}^{n, \lambda}, i_{1}^{n, \lambda}, \ldots, i_{n}^{n, \lambda}\right)$. We can use the same ideas as in [16, Lemma 5.8] to state that the non-negligible terms of the sum must satisfy that for all $n$, there are as much $(n+1)$-tuples involved in $u$ as in $\bar{u}$, which means that

$$
p_{n}+s_{n}=q_{n}+r_{n},
$$

and that we must have the multiset equalities

$$
\begin{aligned}
& \bigcup_{n=1}^{n_{0}}\left\{i_{\mu}^{n, \lambda}, 1 \leq \lambda \leq p_{n}, 0 \leq \mu \leq n\right\}_{m} \cup\left\{\ell_{\mu}^{n, \lambda}, 1 \leq \lambda \leq s_{n}, 0 \leq \mu \leq n\right\}_{m} \\
= & \bigcup_{n=1}^{n_{0}}\left\{j_{\mu}^{n, \lambda}, 1 \leq \lambda \leq q_{n}, 0 \leq \mu \leq n\right\}_{m} \cup\left\{k_{\mu}^{n, \lambda}, 1 \leq \lambda \leq r_{n}, 0 \leq \mu \leq n\right\}_{m} .
\end{aligned}
$$

We deduce that there are a $O\left(N^{\sum_{n} n\left(p_{n}+s_{n}\right)}\right)$ non zero terms in (26) and we can easily show that any subsum over a $o\left(N^{\sum_{n} n\left(p_{n}+s_{n}\right)}\right)$ is negligible so that for now on we shall sum over 
the non pairwise indices. Then, we know that we can neglect any expectation $\mathbb{E}_{\mathbf{U}}$ which won't lead to $\mathrm{Wg}(i d)$ (see (29)) so that (27) becomes

$$
\begin{aligned}
\forall 1 \leq n \leq n_{0}, \quad & \left\{i_{\mu}^{n, \lambda}, 1 \leq \lambda \leq p_{n}, 0 \leq \mu \leq n\right\} \cup\left\{\ell_{\mu}^{n, \lambda}, 1 \leq \lambda \leq s_{n}, 0 \leq \mu \leq n\right\} \\
= & \left\{j_{\mu}^{n, \lambda}, 1 \leq \lambda \leq q_{n}, 0 \leq \mu \leq n\right\} \bigcup\left\{k_{\mu}^{n, \lambda}, 1 \leq \lambda \leq r_{n}, 0 \leq \mu \leq n\right\} .
\end{aligned}
$$

It follows that the set of indices involved in the expansion of the $\operatorname{Tr} \mathbf{A}^{n} \mathbf{N}_{n}, \operatorname{Tr} \mathbf{A}^{-n} \mathbf{N}_{-n}$, $\overline{\operatorname{Tr} \mathbf{A}^{n} \mathbf{N}_{n}}, \overline{\operatorname{Tr} \mathbf{A}^{-n} \mathbf{N}_{-n}}$, is disjoint from the set of indices involved in the expansion of the $\operatorname{Tr} \mathbf{A}^{m} \mathbf{N}_{m}, \operatorname{Tr} \mathbf{A}^{-m} \mathbf{N}_{-m}, \overline{\operatorname{Tr} \mathbf{A}^{m} \mathbf{N}_{m}}, \overline{\operatorname{Tr} \mathbf{A}^{-m} \mathbf{N}_{-m}}$, as long as $n \neq m$. Therefore, Corollary A.4 allows to conclude the proof of $\mathbf{a}$ ).

3.6. Proof of $\mathbf{c})$ : computation of the limit covariance. Let $f, g$ be polynomials in $z$ and $z^{-1}$ and let $\mathbf{M}, \mathbf{N}$ be $N \times N$ deterministic matrices such that, as $N \rightarrow \infty$,

$$
\frac{1}{N} \operatorname{Tr} \mathbf{M} \longrightarrow \tau \quad ; \quad \frac{1}{N} \operatorname{Tr} \mathbf{N} \longrightarrow \tau^{\prime} \quad ; \quad \frac{1}{N} \operatorname{Tr} \mathbf{M N} \mathbf{N}^{*} \longrightarrow \alpha \quad ; \quad \frac{1}{N} \operatorname{Tr} \mathbf{M N} \longrightarrow \beta .
$$

We need to check that the limits of both sequences

$$
\mathbb{E}\left(\operatorname{Tr} f(\mathbf{A}) \mathbf{M}-a_{0}(f) \operatorname{Tr} \mathbf{M}\right)\left(\overline{\operatorname{Tr} g(\mathbf{A}) \mathbf{N}-a_{0}(g) \operatorname{Tr} \mathbf{N}}\right)
$$

and

$$
\mathbb{E}\left(\operatorname{Tr} f(\mathbf{A}) \mathbf{M}-a_{0}(f) \operatorname{Tr} \mathbf{M}\right)\left(\operatorname{Tr} g(\mathbf{A}) \mathbf{N}-a_{0}(g) \operatorname{Tr} \mathbf{N}\right)
$$

are the ones given in the statement of Theorem 2.4. Note that it suffices to compute the limits for $f=g$ and $\mathbf{M}=\mathbf{N}$. Indeed, using the classical polarization identities for $\mathbf{M}$ and $\mathbf{N}$, first for general polynomials $f, g$, we reduce the problem to the case $\mathbf{M}=\mathbf{N}$. Then, we use polarization identities again to reduce the problem to $f=g$.

Also, recall that since $\mathbf{A} \stackrel{\text { law }}{=} e^{i \theta} \mathbf{A}$ for any deterministic $\theta$, we know that for any positive distinct integers $p, q$, we have

$$
\mathbb{E} \operatorname{Tr} \mathbf{A}^{p} \mathbf{M} \operatorname{Tr} \mathbf{A}^{-q} \mathbf{M}=\mathbb{E} \operatorname{Tr} \mathbf{A}^{p} \mathbf{M} \overline{\operatorname{Tr} \mathbf{A}^{q} \mathbf{M}}=0 .
$$

It follows, using (9), (10) and (11), that

$$
\begin{aligned}
\mathbb{E}\left|\operatorname{Tr} f(\mathbf{A}) \mathbf{M}-a_{0}(f) \operatorname{Tr} \mathbf{M}\right|^{2} & =\sum_{\substack{m, n \in \mathbb{Z} \\
\neq 0}} a_{m}(f) \overline{a_{n}(f)} \mathbb{E} \operatorname{Tr} \mathbf{A}^{m} \mathbf{M} \overline{\operatorname{Tr}} \mathbf{A}^{n} \mathbf{M} \\
& =\sum_{n \geq 1}\left(\left|a_{n}(f)\right|^{2} \mathbb{E}\left|\operatorname{Tr} \mathbf{A}^{n} \mathbf{M}\right|^{2}+\left|a_{-n}(f)\right|^{2} \mathbb{E}\left|\operatorname{Tr} \mathbf{A}^{-n} \mathbf{M}\right|^{2}\right) \\
& \longrightarrow \sum_{n \geq 1}\left(\left|a_{n}(f)\right|^{2} b^{2 n}+\left|a_{-n}(f)\right|^{2} a^{-2 n}\right)\left((n-1)|\tau|^{2}+\alpha\right), \\
\mathbb{E}\left(\operatorname{Tr} f(\mathbf{A}) \mathbf{M}-a_{0}(f) \operatorname{Tr} \mathbf{M}\right)^{2} & =\sum_{\substack{m, n \in \mathbb{Z} \\
\neq \neq 0}} a_{m}(f) a_{n}(f) \mathbb{E} \operatorname{Tr} \mathbf{A}^{m} \mathbf{M} \operatorname{Tr} \mathbf{A}^{n} \mathbf{M} \\
& =\sum_{n \geq 1} 2 a_{n}(f) a_{-n}(f) \mathbb{E} \operatorname{Tr} \mathbf{A}^{n} \mathbf{M} \operatorname{Tr} \mathbf{A}^{-n} \mathbf{M}
\end{aligned}
$$


which concludes the proof.

\section{Proof of Corollary 2.14}

It is easy to see that, for any $z \notin S$, we have

$$
\log |\operatorname{det}(z-\mathbf{A})|= \begin{cases}\operatorname{Tr} \log \mathbf{T}+\operatorname{Re} \mathcal{A}_{z}^{N}, & \text { with } \mathcal{A}_{z}^{N}:=\operatorname{Tr} \sum_{n \leq-1} \frac{\mathbf{A}^{n}}{n z^{n}} \text { if }|z|<a . \\ N \log |z|+\operatorname{Re} \mathcal{B}_{z}^{N}, & \text { with } \mathcal{B}_{z}^{N}:=-\operatorname{Tr} \sum_{n \geq 1} \frac{\mathbf{A}^{n}}{n z^{n}} \text { if }|z|>b,\end{cases}
$$

(in the first case, we used the fact that $|\operatorname{det} \mathbf{A}|=\operatorname{det} \mathbf{T}$ ). Then, by Theorem 2.4,

$$
\left(\mathcal{A}_{z}^{N}\right)_{|z|<a} \cup\left(\mathcal{B}_{z}^{N}\right)_{|z|>b}
$$

converges, for the finite-dimensional distributions, to a centered complex Gaussian process

$$
\left(\mathcal{A}_{z}\right)_{|z|<a} \cup\left(\mathcal{B}_{z}\right)_{|z|>b}
$$

with covariance defined by

$$
\begin{aligned}
& \mathbb{E} \mathcal{A}_{z} \mathcal{A}_{z^{\prime}}=0, \quad \mathbb{E} \mathcal{A}_{z} \overline{\mathcal{A}_{z^{\prime}}}=-\log \left(1-\frac{z \overline{z^{\prime}}}{a^{2}}\right), \\
& \mathbb{E} \mathcal{B}_{z} \mathcal{B}_{z^{\prime}}=0, \quad \mathbb{E} \mathcal{B}_{z} \overline{\mathcal{B}_{z^{\prime}}}=-\log \left(1-\frac{b^{2}}{z \overline{z^{\prime}}}\right), \\
& \mathbb{E} \mathcal{A}_{z} \mathcal{B}_{z^{\prime}}=-\log \left(1-\frac{z^{\prime}}{z}\right), \quad \mathbb{E} \mathcal{A}_{z} \overline{\mathcal{B}_{z^{\prime}}}=0
\end{aligned}
$$

where $\log$ denotes the canonical complex log on $B(1,1)$. Then, one concludes by noting that for $A, B \in \mathbb{C}, 2 \operatorname{Re} A \operatorname{Re} B=\operatorname{Re}(A B+A \bar{B})$.

\section{Appendix A.}

A.1. Weingarten calculus on the unitary group. Here we summarize the results we need about integration with respect to the Haar measure on unitary group, (see [25, Cor. 2.4 and Cor. 2.7]).

Let Moeb denote the Möbius function of the lattice of non-crossing partition, defined for example in [44, Lect. 10]. To simplify, for any $k$-tuples $\mathbf{i}=\left(i_{1}, \ldots, i_{k}\right)$ and $\mathbf{j}=\left(j_{1}, \ldots, j_{k}\right)$, we set

$$
u_{\mathbf{i}, \mathbf{j}}:=u_{i_{1} j_{1}} u_{i_{2} j_{2}} \cdots u_{i_{k} j_{k}}
$$


Proposition A.1. Let $k$ be a positive integer and $\mathbf{U}=\left(u_{i j}\right)$ a $N \times N$ Haar-distributed matrix. Let $\mathbf{i}=\left(i_{1}, \ldots, i_{k}\right), \mathbf{i}^{\prime}=\left(i_{1}^{\prime}, \ldots, i_{k}^{\prime}\right), \mathbf{j}=\left(j_{1}, \ldots, j_{k}\right)$ and $\mathbf{j}^{\prime}=\left(j_{1}^{\prime}, \ldots, j_{k}^{\prime}\right)$ be four $k$-tuples of $\{1, \ldots, N\}$. Then

$$
\mathbb{E}\left[u_{\mathbf{i}, \mathbf{j}} \overline{u_{\mathbf{i}^{\prime}, \mathbf{j}^{\prime}}}\right]=\sum_{\sigma, \tau \in S_{k}} \delta_{i_{1}, i_{\sigma(1)}^{\prime}} \ldots \delta_{i_{k}, i_{\sigma(k)}^{\prime}} \delta_{j_{1}, j_{\tau(1)}^{\prime}} \ldots \delta_{j_{k}, j_{\tau(k)}^{\prime}} \mathrm{Wg}\left(\tau \sigma^{-1}\right),
$$

where $\mathrm{Wg}$ is a function called the Weingarten function. Moreover, for $\sigma \in S_{k}$, the asymptotical behavior of $\mathrm{Wg}(\sigma)$ is given by

$$
N^{k+|\sigma|} \operatorname{Wg}(\sigma)=\operatorname{Moeb}(\sigma)+O\left(\frac{1}{N^{2}}\right),
$$

where $|\sigma|$ denotes the minimal number of factors necessary to write $\sigma$ as a product of transpositions.

Remark A.2. One should notice that if all $k$-tuples $\left(i_{1}, \ldots, i_{k}\right),\left(j_{1}, \ldots, j_{k}\right),\left(i_{1}^{\prime}, \ldots, i_{k}^{\prime}\right)$, and $\left(j_{1}^{\prime}, \ldots, j_{k}^{\prime}\right)$ have pairwise distinct entries, then (28) becomes simpler because in this case there is at most one non-zero term in the sum.

Remark A.3. The permutation $\sigma$ for which $\operatorname{Wg}(\sigma)$ will have the largest order is the only one satisfying $|\sigma|=0$, i.e. $\sigma=i d$. Also, $\operatorname{Moeb}(i d)=1$ (see [25]).

Here is a useful corollary which permits to simplify many computations.

Corollary A.4. Let $\mathbf{i}=\left(i_{1}, \ldots, i_{p}\right), \mathbf{j}=\left(j_{1}, \ldots, j_{p}\right), \mathbf{k}=\left(k_{1}, \ldots, k_{q}\right), \mathbf{l}=\left(\ell_{1}, \ldots, \ell_{q}\right)$, $\mathbf{i}^{\prime}=\left(i_{1}^{\prime}, \ldots, i_{p}^{\prime}\right), \mathbf{j}^{\prime}=\left(j_{1}^{\prime}, \ldots, j_{p}^{\prime}\right), \mathbf{k}^{\prime}=\left(k_{1}^{\prime}, \ldots, k_{q}^{\prime}\right), \mathbf{l}^{\prime}=\left(\ell_{1}^{\prime}, \ldots, \ell_{q}^{\prime}\right)$ be tuples such that the multisets defined by $\mathbf{i}$ and $\mathbf{i}^{\prime}$ (resp. by $\mathbf{j}$ and $\mathbf{j}^{\prime}$, by $\mathbf{k}$ and $\mathbf{k}^{\prime}$, by $\mathbf{l}$ and $\mathbf{l}^{\prime}$ ) are equal and such that

Then

$$
\left\{i_{1}, \ldots, i_{p}\right\} \cap\left\{k_{1}, \ldots, k_{q}\right\}=\left\{j_{1}, \ldots, j_{p}\right\} \cap\left\{l_{1}, \ldots, l_{q}\right\}=\emptyset .
$$

$$
\mathbb{E}\left[u_{\mathbf{i}, \mathbf{j}} u_{\mathbf{k}, \mathbf{l}} \overline{u_{\mathbf{i}^{\prime}, \mathbf{j}^{\prime}} u_{\mathbf{k}^{\prime}, \mathbf{l}^{\prime}}}\right]=\mathbb{E}\left[u_{\mathbf{i}, \mathbf{j}} \overline{u_{\mathbf{i}^{\prime}, \mathbf{j}^{\prime}}}\right] \mathbb{E}\left[u_{\mathbf{k}, \mathbf{l}} \overline{u_{\mathbf{k}^{\prime}, \mathbf{l}^{\prime}}}\right]\left(1+O\left(\frac{1}{N^{2}}\right)\right)
$$

Proof. To prove this result, we first recall the exact expression of the Möbius function : for any permutation $\sigma$ with cycle decomposition $C_{1} C_{2} \cdots C_{r}$,

$$
\operatorname{Moeb}(\sigma)=\prod_{i=1}^{r}(-1)^{\left|C_{i}\right|-1} \operatorname{Cat}_{\left|C_{i}\right|-1},
$$

where Cat $_{k}$ is the $k$-th Catalan number, $\frac{1}{k+1}\left(\begin{array}{c}2 k \\ k\end{array}\right)$. Then, obviously, if $\sigma$ and $\tau$ are two permutations with disjoint supports, then

$$
\operatorname{Moeb}(\sigma \circ \tau)=\operatorname{Moeb}(\sigma) \operatorname{Moeb}(\tau) \quad \text { and } \quad|\sigma \circ \tau|=|\sigma|+|\tau| \text {. }
$$

Thus

$$
N^{p+q+|\sigma \circ \tau|} \operatorname{Wg}(\sigma \circ \tau)=\operatorname{Moeb}(\sigma \circ \tau)+O\left(\frac{1}{N^{2}}\right)
$$




$$
\begin{aligned}
& =\operatorname{Moeb}(\sigma) \operatorname{Moeb}(\tau)+O\left(\frac{1}{N^{2}}\right) \\
& =n^{p+|\sigma|} \operatorname{Wg}(\sigma) N^{q+|\tau|} \operatorname{Wg}(\tau)\left(1+O\left(\frac{1}{N^{2}}\right)\right)
\end{aligned}
$$

So that

$$
\mathrm{Wg}(\sigma \circ \tau)=\operatorname{Wg}(\sigma) \operatorname{Wg}(\tau)\left(1+O\left(\frac{1}{N^{2}}\right)\right) .
$$

One can easily conclude.

We also need the following lemmas in the paper.

Lemma A.5. Let $\mathbf{A}=\mathbf{U T}$ with $\mathbf{U}$ Haar-distributed on the unitary group and $\mathbf{T}$ deterministic. Let $\varepsilon>0$. There is a finite constant $C$ depending only on $\varepsilon$ (in particular, independent of $N$ and of $\mathbf{T})$ such that for all positive integer $n$ such that $n^{6}<(2-\varepsilon) N$, we have

$$
\left.\mathbb{E} \operatorname{Tr} \mathbf{A}^{n}\left(\mathbf{A}^{n}\right)^{*}\right) \leq C N n^{2}\left(m_{2}+\frac{n m_{\infty}^{2}}{N}\right)^{n}
$$

and

$$
\mathbb{E}\left[\left|\operatorname{Tr} \mathbf{A}^{n}\right|^{2}\right] \leq C\left(m_{2}+\frac{n m_{\infty}^{2}}{N}\right)^{n}
$$

where $m_{2}:=N^{-1} \operatorname{Tr} \mathbf{T T}^{*}$ and $m_{\infty}:=\|\mathbf{T}\|_{\mathrm{op}}$.

Proof. See [11, Th. 1].

Lemma A.6. Let $\mathbf{V}$ be Haar-distributed and let $\mathbf{A}, \mathbf{B}, \mathbf{C}, \mathbf{D}$ be deterministic $N \times N$ matrices. Then we have

$$
\begin{aligned}
& \mathbb{E} \operatorname{Tr} \mathbf{A V B V}^{*} \operatorname{Tr} \mathbf{C V D V}^{*}=\frac{1}{N^{2}-1}(\operatorname{Tr} \mathbf{A} \operatorname{Tr} \mathbf{C} \operatorname{Tr} \mathbf{B} \operatorname{Tr} \mathbf{D}+\operatorname{Tr} \mathbf{A C} \operatorname{Tr} \mathbf{B D}) \\
& -\frac{1}{N\left(N^{2}-1\right)}(\operatorname{Tr} \mathbf{A} \operatorname{Tr} \mathbf{C} \operatorname{Tr} \mathbf{B D}+\operatorname{Tr} \mathbf{A C} \operatorname{Tr} \mathbf{B} \operatorname{Tr} \mathbf{D})
\end{aligned}
$$

Proof. Let $\mathcal{M}_{N}(\mathbb{C})$ denote the set of $N \times N$ complex matrices. It has already been proved, in $\left[16\right.$, Lem. 5.9], that for any matrices $\mathbf{A}, \mathbf{B}, \mathbf{C}, \mathbf{D} \in \mathcal{M}_{N}(\mathbb{C})$, we have

$$
\begin{aligned}
\mathbb{E} \operatorname{Tr} \mathbf{A V B V} \mathbf{C V D V}^{*}= & \frac{1}{N^{2}-1}\{\operatorname{Tr} \mathbf{A C} \operatorname{Tr} \mathbf{B} \operatorname{Tr} \mathbf{D}+\operatorname{Tr} \mathbf{A} \operatorname{Tr} \mathbf{C} \operatorname{Tr} \mathbf{B D}\} \\
& -\frac{1}{N\left(N^{2}-1\right)}\{\operatorname{Tr} \mathbf{A C} \operatorname{Tr} \mathbf{B D}+\operatorname{Tr} \mathbf{A} \operatorname{Tr} \mathbf{C} \operatorname{Tr} \mathbf{B} \operatorname{Tr} \mathbf{D}\} .
\end{aligned}
$$

We deduce that

$$
\mathbb{E} \mathbf{V} \otimes \mathbf{V}^{*} \otimes \mathbf{V} \otimes \mathbf{V}^{*}=\frac{1}{N^{2}-1}\left\{\sum_{i, j, k, \ell} E_{j, k} \otimes E_{k, j} \otimes E_{i, \ell} \otimes E_{\ell, i}+E_{i, j} \otimes E_{k, \ell} \otimes E_{\ell, k} \otimes E_{j, i}\right\}
$$




$$
-\frac{1}{N\left(N^{2}-1\right)}\left\{\sum_{i, j, k, \ell} E_{j, k} \otimes E_{\ell, j} \otimes E_{i, \ell} \otimes E_{k, i}+E_{i, j} \otimes E_{j, k} \otimes E_{k, \ell} \otimes E_{\ell, i}\right\},
$$

where the $E_{r, s}$ denote the elementary matrices. Indeed, the linear morphism $\Psi$ from $\mathcal{M}_{N}(\mathbb{C})^{\otimes 4}$ to the space of 4-linear forms on $\mathcal{M}_{N}(\mathbb{C})$ defined by

$$
\Psi(\mathbf{M} \otimes \mathbf{N} \otimes \mathbf{P} \otimes \mathbf{Q})(\mathbf{A}, \mathbf{B}, \mathbf{C}, \mathbf{D}):=\operatorname{Tr} \mathbf{A M B N C P D Q}
$$

is an isomorphism, and (32) proves that the left and right hand terms of (33) have the same image by $\Psi$. Then, applying

$$
\mathbf{M} \otimes \mathbf{N} \otimes \mathbf{P} \otimes \mathbf{Q} \longmapsto \operatorname{Tr}(\mathbf{A M B N}) \operatorname{Tr}(\mathbf{C P D Q})
$$

on both sides of (33), we deduce the lemma.

A.2. Moments of a Gaussian vector with values in $\mathbb{C}^{2}$. The following lemma allows to prove that a complex random vector $(\tilde{X}, \tilde{Y})$ is Gaussian without having to compute all its joint moments, by only proving an induction relation.

Lemma A.7. Let $(X, Y)$ be a Gaussian random vector with values in $\mathbb{C}^{2}$ whose distribution is characterized by

$$
\mathbb{E} X=\mathbb{E} X^{2}=\mathbb{E} Y=\mathbb{E} Y^{2}=0,
$$

and

$$
\mathbb{E}|X|^{2}=\sigma_{X} ; \mathbb{E}|Y|^{2}=\sigma_{Y} ; \mathbb{E} X Y=\sigma_{X Y} ; \mathbb{E} X \bar{Y}=0
$$

Then, the moments

$$
m_{p, q, r, s}:=\mathbb{E} X^{p} \bar{X}^{q} Y^{r} \bar{Y}^{s}
$$

satisfy

$$
\begin{gathered}
p-q \neq r-s \Longrightarrow m_{p, q, r, s}=0 \\
m_{p, q, r, s}=\left\{\begin{array}{l}
q \sigma_{X} m_{p-1, q-1, r, s}+r \sigma_{X Y} m_{p-1, q, r-1, s} \\
p \sigma_{X} m_{p-1, q-1, r, s}+s \overline{\sigma_{X Y}} m_{p, q-1, r, s-1} \\
s \sigma_{Y} m_{p, q, r-1, s-1}+p \sigma_{X Y} m_{p-1, q, r-1, s} \\
r \sigma_{Y} m_{p, q, r-1, s-1}+q \bar{\sigma}_{X Y} m_{p, q-1, r, s-1}
\end{array}\right.
\end{gathered}
$$

and

$$
m_{p, 0, p, 0}=\mathbb{E}(X Y)^{p}=p ! \sigma_{X Y}^{p}
$$

Conversely, if $(\tilde{X}, \tilde{Y})$ is a random vector with values in $\mathbb{C}^{2}$ such that both $\tilde{X}$ and $\tilde{Y}$ are Gaussian and have joint moments $\tilde{m}_{p, q, r, s}$ satisfying (34), (35), (37), (38) and (39), then $(\tilde{X}, \tilde{Y})$ is Gaussian. 
Proof. First, one easily obtains (37) by noticing that for any $\theta \in \mathbb{R},\left(\mathrm{e}^{\mathrm{i} \theta} X, \mathrm{e}^{-\mathrm{i} \theta} Y\right) \stackrel{\text { law }}{=}(X, Y)$. To prove the remaining, we consider $(X, Y)$ as a real 4 -tuple $(\Re(X), \Im(X), \Re(Y), \Im(Y))=$ $\left(x_{1}, x_{2}, x_{3}, x_{4}\right)$ with covariance matrix

$$
\Gamma:=\frac{1}{2}\left(\begin{array}{cccc}
\sigma_{X} & 0 & \Re\left(\sigma_{X Y}\right) & \Im\left(\sigma_{X Y}\right) \\
0 & \sigma_{X} & \Im\left(\sigma_{X Y}\right) & -\Re\left(\sigma_{X Y}\right) \\
\Re\left(\sigma_{X Y}\right) & \Im\left(\sigma_{X Y}\right) & \sigma_{Y} & 0 \\
\Im\left(\sigma_{X Y}\right) & -\Re\left(\sigma_{X Y}\right) & 0 & \sigma_{Y}
\end{array}\right)
$$

Its Fourier transform is given, for $\mathbf{t}=\left(t_{1}, t_{2}, t_{3}, t_{4}\right)$, by

$$
\begin{aligned}
\Phi(\mathbf{t}) & :=\mathbb{E} \exp \left(\mathrm{i}\left(t_{1} x_{1}+t_{2} x_{2}+t_{3} x_{3}+t_{4} x_{4}\right)\right) \\
& =\exp \left\{-\frac{1}{4}\left(\sigma_{X}\left(t_{1}^{2}+t_{2}^{2}\right)+\sigma_{Y}\left(t_{3}^{2}+t_{4}^{2}\right)\right)-\frac{1}{2} \Re\left(\sigma_{X Y}\right)\left(t_{1} t_{3}-t_{2} t_{4}\right)-\frac{1}{2} \Im\left(\sigma_{X Y}\right)\left(t_{2} t_{3}+t_{1} t_{4}\right)\right\}
\end{aligned}
$$

We define the differential operators

$$
\begin{array}{ll}
\partial_{X}=\partial_{1}+\mathrm{i} \partial_{2} & \partial_{\bar{X}}=\partial_{1}-\mathrm{i} \partial_{2} \\
\partial_{Y}=\partial_{3}+\mathrm{i} \partial_{4} & \partial_{\bar{Y}}=\partial_{3}-\mathrm{i} \partial_{4}
\end{array}
$$

so that

$$
\mathbb{E} X^{p} \bar{X}^{q} Y^{r} \bar{Y}^{s}=\left.(-\mathrm{i})^{p+q+r+s} \partial_{X}^{p} \partial_{\bar{X}}^{q} \partial_{Y}^{r} \partial_{\bar{Y}}^{s} \Phi(\mathbf{t})\right|_{\mathbf{t}=0}
$$

and

$$
\begin{aligned}
& \partial_{X} \Phi(\mathbf{t})=-\frac{1}{2}\left(\sigma_{X}\left(t_{1}+\mathrm{i} t_{2}\right)+\sigma_{X Y}\left(t_{3}-\mathrm{i} t_{4}\right)\right) \Phi(\mathbf{t}) \\
& \partial_{\bar{X}} \Phi(\mathbf{t})=-\frac{1}{2}\left(\sigma_{X}\left(t_{1}-\mathrm{i} t_{2}\right)+\overline{\sigma_{X Y}}\left(t_{3}+\mathrm{i} t_{4}\right)\right) \Phi(\mathbf{t}) \\
& \partial_{Y} \Phi(\mathbf{t})=-\frac{1}{2}\left(\sigma_{Y}\left(t_{3}+\mathrm{i} t_{4}\right)+\sigma_{X Y}\left(t_{1}-\mathrm{i} t_{2}\right)\right) \Phi(\mathbf{t}) \\
& \partial_{\bar{Y}} \Phi(\mathbf{t})=-\frac{1}{2}\left(\sigma_{Y}\left(t_{3}-\mathrm{i} t_{4}\right)+\overline{\sigma_{X Y}}\left(t_{1}+\mathrm{i} t_{2}\right)\right) \Phi(\mathbf{t})
\end{aligned}
$$

We easily deduce that

$$
\left.\partial_{X}^{p} \partial_{\bar{X}}^{q} \partial_{Y}^{r} \partial_{\bar{Y}}^{s} \Phi(\mathbf{t})\right|_{\mathbf{t}=0}=-\left.q \sigma_{X} \partial_{X}^{p-1} \partial_{\bar{X}}^{q-1} \partial_{Y}^{r} \partial_{\bar{Y}}^{s} \Phi(\mathbf{t})\right|_{\mathbf{t}=0}-\left.r \sigma_{X Y} \partial_{X}^{p-1} \partial_{\bar{X}}^{q} \partial_{Y}^{r-1} \partial_{\bar{Y}}^{s} \Phi(\mathbf{t})\right|_{\mathbf{t}=0}
$$

so that

$$
\mathbb{E} X^{p} \bar{X}^{q} Y^{r} \bar{Y}^{s}=q \sigma_{X} \mathbb{E} X^{p-1} \bar{X}^{q-1} Y^{r} \bar{Y}^{s}+r \sigma_{X Y} \mathbb{E} X^{p-1} \bar{X}^{q} Y^{r-1} \bar{Y}^{s}
$$

This proves (38). To prove the last point, we simply write

$$
\mathbb{E}(X Y)^{p}=\left.(-1)^{p} \partial_{X}^{p} \partial_{Y}^{p} \Phi(\mathbf{t})\right|_{\mathbf{t}=0} \quad ; \quad \partial_{X}^{p} \Phi(\mathbf{t})=\left(-\frac{\sigma_{X}\left(t_{1}+\mathrm{i} t_{2}\right)+\sigma_{X Y}\left(t_{3}-\mathrm{i} t_{4}\right)}{2}\right)^{p} \Phi(\mathbf{t}) .
$$


Then, using Leibniz formula and noticing that for all $k \leq p$,

$$
\left.\partial_{Y}^{k}\left(-\frac{\sigma_{X}\left(t_{1}+\mathrm{i} t_{2}\right)+\sigma_{X Y}\left(t_{3}-\mathrm{i} t_{4}\right)}{2}\right)^{p}\right|_{\mathbf{t}=0}=\left\{\begin{array}{l}
0 \quad \text { if } k<p \\
p !\left(\sigma_{X Y}\right)^{p} \text { if } k=p
\end{array},\right.
$$

one can easily conclude.

Conversely, let $(\tilde{X}, \tilde{Y})$ be a random vector with values in $\mathbb{C}^{2}$ such that both $\tilde{X}$ and $\tilde{Y}$ are Gaussian and have joint moments $\tilde{m}_{p, q, r, s}$ satisfying (34), (35), (37), (38) and (39). Let $\mathbb{N}$ denote the set of non-negative integers and let us define the sets

$$
\begin{gathered}
\mathcal{K}:=\left\{(p, q, r, s) \in \mathbb{N}^{4} ; p-q \neq r-s\right\}, \\
\mathcal{H}_{0}:=\left\{(p, q, r, s) \in \mathbb{N}^{4} ;(r+s+|p-q|)(p+q+|r-s|)(p+r+|q-s|)(q+s+|p-r|)=0\right\}
\end{gathered}
$$

and, for $k \geq 1$,

$$
\begin{aligned}
\mathcal{H}_{k}:=\left\{(p, q, r, s) \in \mathbb{N}^{4} ;\right. & (p-1, q-1, r, s) \text { or }(p-1, q, r-1, s) \text { or } \\
& \left.(p, q-1, r, s-1) \text { or }(p, q, r-1, s-1) \in \mathcal{H}_{k-1}\right\}
\end{aligned}
$$

Then by hypothesis, the joint moments function $\tilde{m}_{p, q, r, s}$ coincides with $m_{p, q, r, s}$ on $\mathcal{K} \cup \mathcal{H}_{0}$. Besides, by (38), if $\tilde{m}_{p, q, r, s}$ coincides with $m_{p, q, r, s}$ on $\mathcal{H}_{k-1}$, then $\tilde{m}_{p, q, r, s}$ coincides with $m_{p, q, r, s}$ on $\mathcal{H}_{k}$. As

$$
\mathbb{N}^{4}=\mathcal{K} \cup \bigcup_{k \geq 0} \mathcal{H}_{k}
$$

we deduce that $\tilde{m}_{p, q, r, s}$ coincides with $m_{p, q, r, s}$ on $\mathbb{N}^{4}$, wich implies that $(\tilde{X}, \tilde{Y}) \stackrel{\text { law }}{=}(X, Y)$.

Acknowledgments. The authors wish to thank the anonymous referee for his careful reading and his useful advices.

\section{REFERENCES}

[1] G. Anderson, O. Zeitouni A CLT for a band matrix model, Probab. Theory Rel. Fields, 2005, 134, 283-338

[2] G. Anderson, A. Guionnet, O. Zeitouni An Introduction to Random Matrices. Cambridge studies in advanced mathematics, 118 (2009).

[3] A. D'Aristotile, P. Diaconis, C. Newman Brownian motion and the classical groups. With Probability, Statisitica and their applications: Papers in Honor of Rabii Bhattacharaya. Edited by K. Athreya et al. 97-116. Beechwood, OH: Institute of Mathematical Statistics, 2003.

[4] Z.D. Bai, J. Silverstein CLT for linear spectral statistics of large-dimensional sample covariance matrices. Ann. Probab. 32, 2004, 533-605.

[5] Z.D. Bai, X. Wang, W. Zhou CLT for linear spectral statistics of Wigner matrices. Electron. J. Probab. 14 (2009), no. 83, 2391-2417.

[6] Z.D. Bai, J. Yao On the convergence of the spectral empirical process of Wigner matrices. Bernoulli 11 (2005) 1059-1092.

[7] J. Baik, G. Ben Arous, S. Péché Phase transition of the largest eigenvalue for nonnull complex sample covariance matrices. Ann. Probab., 33(5):1643-1697, 2005. 
[8] A. Basak, A. Dembo Limiting spectral distribution of sums of unitary and orthogonal matrices. Electron. Commun. Probab. 18 (2013), no. 69, 19 pp.

[9] S.R. Bell The Cauchy transform, potential theory, and conformal mapping. (1992) Studies in advanced Mathematics. CRC Press, Boca Raton, FL.

[10] F. Benaych-Georges Central limit theorems for the Brownian motion on large unitary groups, Bull. Soc. Math. France,Vol. 139, no. 4 (2011) 593-610.

[11] F. Benaych-Georges Exponential bounds for the support convergence in the Single Ring Theorem, J. Funct. Anal. 268 (2015), pp. 3492-3507.

[12] F. Benaych-Georges, G. Cébron, J. Rochet Fluctuation of matrix entries and application to outliers of elliptic matrices, arXiv:1602.02929.

[13] F. Benaych-Georges, A. Guionnet, C. Male Central limit theorems for linear statistics of heavy tailed random matrices. Comm. Math. Phys. Vol. 329 (2014), no. 2, 641-686.

[14] F. Benaych-Georges, R.N. Rao The eigenvalues and eigenvectors of finite, low rank perturbations of large random matrices, Adv. Math. (2011), Vol. 227, no. 1, 494-521.

[15] F. Benaych-Georges, R.N. Rao The singular values and vectors of low rank perturbations of large rectangular random matrices, J. Multivariate Anal., Vol. 111 (2012), 120-135.

[16] F. Benaych-Georges, J. Rochet Outliers in the Single Ring Theorem, Probab. Theory Rel. Fields, Vol. 165 (2016), no. 1, 313-363.

[17] C. Bordenave, M. Capitaine Outlier eigenvalues for deformed i.i.d. random matrices. Comm. Pure Appl. Math. 69 (2016), no. 11, 2131-2194.

[18] C. Bordenave, D. Chafaï Around the circular law, Probab. Surv. 9 (2012), 1-89.

[19] É. Borel Sur les principes de la théorie cinétique des gaz. Annales de l'École Normale Supérieure 23 (1906), 9-32.

[20] M. Capitaine, C. Donati-Martin, D. Féral, M. Février Free convolution with a semi-circular distribution and eigenvalues of spiked deformations of Wigner matrices, Electron. J. Prob. Vol. 16 (2011), $1750-1792$.

[21] G. Cébron, T. Kemp Fluctuations of Brownian Motions on $\mathbb{G L}_{N}$. arXiv:1409.5624.

[22] S. Chatterjee Fluctuations of eigenvalues and second order Poincaré inequalities, Probab. Theory Related Fields, 143, 2009,1-40.

[23] S. Chatterjee, E. Meckes Multivariate normal approximation using exchangeable pairs ALEA 4 (2008).

[24] B. Collins, J.A. Mingo, P. Śniady, R. Speicher Second order freeness and fluctuations of random matrices. III. Higher order freeness and free cumulants. Doc. Math. 12 (2007), 1-70.

[25] B. Collins, P. Sniady Integration with respect to the Haar measure on unitary, orthogonal and symplectic group. Comm. Math. Phys., 264(3):773-795, 2006.

[26] B. Collins, M. Stolz Borel theorems for random matrices from the classical compact symmetric spaces. Ann. Probab. Vol. 36, no. 3 (2008), 876-895.

[27] P. Diaconis, M. Shahshahani On the eigenvalues of random matrices, Studies in applied probability, J. Appl. Probab. 31A (1994), 49-62.

[28] P. Diaconis, S. Evans Linear functionals of eigenvalues of random matrices, Trans. Amer. Math. Soc., 353, 2001, 2615-2633.

[29] B. Duplantier, R. Rhodes, S. Sheffield, V. Vargas Log-correlated Gaussian fields: an overview, arXiv.

[30] V.L. Girko The elliptic law, Teoriya Veroyatnostei i ee Primeneniya, 30(4):640-651, 1985.

[31] A. Guionnet, M. Krishnapur, O. Zeitouni The Single Ring Theorem. Ann. of Math. (2) 174 (2011), no. 2, 1189-1217.

[32] A. Guionnet, O. Zeitouni Support convergence in the Single Ring Theorem. Probab. Theory Related Fields 154 (2012), no. 3-4, 661-675.

[33] K. Johansson On the fluctuations of eigenvalues of random Hermitian matrices. Duke Math. J. 91 1998, 151-204. 
[34] D. Jonsson Some limit theorems for the eigenvalues of a sample covariance matrix. J. Mult. Anal. 12, 1982, 1-38.

[35] T. Jiang How many entries of a typical orthogonal matrix can be approximated by independent normals? Ann. Probab. 34(4), 1497-1529. 2006.

[36] T. Kemp Heat Kernel Empirical Laws on $\mathbb{U}_{N}$ and $\mathbb{G L}_{N}$. J. Theor. Probab (2015). doi:10.1007/s10959015-0643-7.

[37] A. M. Khorunzhy, B. A. Khoruzhenko, L. A. Pastur Asymptotic properties of large random matrices with independent entries, J. Math. Phys. 37 (1996) 5033-5060.

[38] T. Lévy, M. Maïda Central limit theorem for the heat kernel measure on the unitary group. J. Funct. Anal. 259 (2010), no. 12, 3163-3204.

[39] A. Lytova, L. Pastur Central limit theorem for linear eigenvalue statistics of random matrices with independent entries, Ann. Probab., 37, 2009, 1778-1840.

[40] E. Meckes Linear functions on the classical matrix groups. Trans. Amer. Math. Soc. 360 (2008), no. $10,5355-5366$.

[41] J.A. Mingo, A. Nica Annular noncrossing permutations and partitions, and second-order asymptotics for random matrices. Int. Math. Res. Not. 2004, no. 28, 1413-1460.

[42] J.A. Mingo, R. Speicher Second order freeness and fluctuations of random matrices. I. Gaussian and Wishart matrices and cyclic Fock spaces. J. Funct. Anal. 235 (2006), no. 1, 226-270.

[43] J.A. Mingo, P. Śniady, R. Speicher, Second order freeness and fluctuations of random matrices. II. Unitary random matrices. Adv. Math. 209 (2007), no. 1, 212-240.

[44] A. Nica, R. Speicher Lectures on the combinatorics of free probability. London Mathematical Society Lecture Note Series, 335. Cambridge University Press, Cambridge, 2006.

[45] S. O'Rourke, D. Renfrew Low rank perturbations of large elliptic random matrices, Electron. J. Probab. 19 (2014), no. 43, 65 pp.

[46] S. O'Rourke, D. Renfrew Central limit theorem for linear eigenvalue statistics of elliptic random matrices, to appear in J. Theoret. Probab.

[47] S. O'Rourke, D. Renfrew, A. Soshnikov On fluctuations of matrix entries of regular functions of Wigner matrices with non-identically distributed entries. J. Theoret. Probab. 26 (2013), no. 3, 750780.

[48] S. Péché The largest eigenvalue of small rank perturbations of Hermitian random matrices, Prob. Theory Relat. Fields, 134 127-173, 2006.

[49] Y. Peres, B. Virág Zeros of the i.i.d. Gaussian power series: a conformally invariant determinantal process. Acta. Math. 194. (2005) $1-35$.

[50] A. Pizzo, D. Renfrew, A. Soshnikov Fluctuations of matrix entries of regular functions of Wigner matrices. J. Stat. Phys. 146 (2012), no. 3, 550-591.

[51] B. Rider, J. Silverstein Gaussian fluctuations for non-Hermitian random matrix ensembles. Ann. Probab. 34 (2006), no. 6, 2118-2143.

[52] B. Rider, B. Virág The noise in the circular law and the Gaussian free field. Int. Math. Res. Not. IMRN 2007, no. 2.

[53] M. Shcherbina Central Limit Theorem for Linear Eigenvalue Statistics of the Wigner and Sample Covariance Random Matrices, Journal of Mathematical Physics, Analysis, Geometry, 7(2), (2011), 176-192.

[54] Y. Sinai, A. Soshnikov Central limit theorem for traces of large random symmetric matrices with independent matrix elements, Bol. Soc. Brasil. Mat. (N.S.), 29, 1998, 1-24.

[55] A. Soshnikov The central limit theorem for local linear statistics in classical compact groups and related combinatorial identities, Ann. Probab., 28 (2000), 1353-1370.

[56] T. Tao Outliers in the spectrum of i.i.d. matrices with bounded rank perturbations. Probab. Theory Related Fields 155 (2013), no. 1-2, 231-263. 